\title{
Conferences and Schools
}

\section{Guide to Entries}

In 1991, Europhysics News is to publish two Meetings Issues: in March and November. Urgent up-datings will also be included in the July Directory Issue in the form of advertisements charged at SFR 100.- per insertion.

The events listed here are those for which details were received at the EPS Secretariat before 28 February 1991. In general, all events interesting European physicists which take place from the middle of April 1991 are cited. For events prior to that date readers are referred to the Nov. 1990 issue.

The listings are in date order divided into two categories - Conferences and Schools although the distinction between these two categories is not always evident. Europhysics Conferences, i.e. meetings organized by EPS, its Divisions and Sections, have titles printed in bold type and are

\section{Conferences 1991}

\section{May / June}

London, UK

A. Gevers, EWGIT, POB 2, LN-1755 ZG Petten $++31(2246) 5656 / 1002$

May / June

Minsk, USSR

10th Workshop: Gravitation and Electromagnetism

G.V. Shishkin, Theoretical Physics Dept., Bielorussian Unishkin, Theoretical Physics Dept., Biel

1 - 3 May Eindhoven, The Netherlands Eindhoven, The

\section{Microelectronics}

W. van Haeringen, Congress Office

Eindhoven Univ of Technology

POB 513, NL-5600 MB

$++31(40) 474000 / 463120$

Bitnet: :tnnidl @ heitue5

A: 1 Mar 91 / Ab: 15 Jan 91 / PP / FLR 275.-

6
6

11th World Meterological Cong.

OMM, 41, Giuseppe-Motta, CP 5, CH-1211 Geneva 20 $++41(22) 346400 /$ -

$5-11$

3rd European Crystal Growth Conf.

Budapest, Hungary

E. Lendvay, Res. Inst. for Technical Physics, Hungarian Acad. of Sci., POB 76, H-1325 Budapest $\mathrm{IUCr}$

6 - 10

Trieste, Italy

5th Workshop on Perspectives in Nuclear Physics at Intermediate Energies

Int. Centre for Theo. Physics, POB 586, 1-34100 Trieste ++39 (40) $22401 / 224163$

$$
\text { limited / INFN, SISSA }
$$

6 - 10

Rome, Italy

3rd Int. Conf. on the Formation of Semiconductor Interfaces

P. Perfetti, INFN del CNR, via E. Fermi, 38 1.00044 Frascati

++39 (396) $9426335 /$

$6-9$

San Francisco, CA, USA

14th Biennial IEEE Particle Accelerator Conference

M. Allen, POB 4349, Bin 68, Stanford, CA 94309, USA ++1 (415) $9262820 / 9263564$ matallen@slacvm

Ab: 3 Dec 90 / PP / IEEE, Nucl. Plasma Sci. Soc., APS, AFOSR

9- 11

Bunsentagung 1991: Thin Layers

Bochum, Germany

H. Behret, Duetsch Bunsen-Ges. f. Physikalische Chemie, Varrentrappstr. 40-42

W-6000 Frankfurt/Main 90

++ (69) $7917201 / 7917322$

Ab: 4 Apr 91 / PP / German / DM 130.10. - students

$13-15$ shop on Mic

Alpbach, Austria Techniques

CCT, 1547, ch. Merivale, Ottawa, Ontario, K1A OY 7, CA $++1(613) 952050 / 9527353$ boxed. Events sponsored by EPS have titles printed in bold type; Europhysics Study Conferences have an E preceding the date; European Research Conferences in Physics organized in cooperation with the ESF, Strasbourg are boxed.

For inclusion in the November 1991 issue, organizers should send details to the EPS Secretariat by 15 October 1991 at the latest. Even if full details are not yet available, the preliminary information should be given.

Entries are selected from a database compiled using information supplied by organizers and by EPS meetings correspondents. Partial listings of the complete database are available.

Application for sponsorship of a meeting should be made to the EPS Secretariat in Geneva as early as possible.

\section{Key to Abbreviations}

A: Application deadline

Ab: Abstracts deadline

inv.: restricted to invited applicants

NP: no proceedings will be published

$P$ : Papers deadline (information on proceedings has not been received)

PP: proceedings will be published.

++ : country (area) tel. / fax

email - bitnet address or other

The information is given in this order:

Date

Venue

Title

Contact for information

A: .... / Ab: .... / PP, NP or P: Papers deadline / Number of participants / inv. / languages other than English / Conference fee / Sponsorship.

after the date indicates an important amendment to the last published entry.

\section{3 - 15 May}

Berlin, Germany

Annual Meeting of the German Society of Rheolog

K. Kirscheke, Deutsche Rheologische Ges. e.V Unter den Eichen 87, W-1000 Berlin 45 ++49 (3) $81045209 / 8112029$

A: 1 Dec $90 /$ Ab: 1 Dec 90/PP: 15 Apr $91 / 150$ German / DM 250.-; 200. - IOM's; students free $13-16$ students free
Trieste, Italy

Trieste Conf. on Quantum Field Theory and Condensed Matter Physics

Int. Centre for Theo. Physics, POB 586, I-34100 Trieste ++39 (40) $22401 / 224163$

13 - 17 Dresden, Germany

5 th Meeting on Nuclear Analytical Methods

G. Loos, Zentralinst. f. Kernforschung Rossendorf, Postfach 19, 0-8051 Dresden

$13-17$

Jülich, Germany

Int. CODATA Conf. on Nuclear Data for Science and Technology

Tagungsburo, KFA Jülich, Postfach 1913 W-5170 Jülich

- I++49 (2461) 613833 Ab: 15 Oct $90 /$ DM 400 . -

$13-17$

Baltimore, MD, USA

QELS '91: Quantum Electronics Laser Science Conf. Optical Society of America, 2010 Massachusetts Ave., Washington D.C. 20036, USA ++1 (202) $2238130 / 2231096$ PP: 17 Apr 91 / APS, IEEE, OSA

$14-18$

Dubrovnik, Yugoslavia

2nd European Workshop on Modern Developments \& Applications in Microbeam Analysis

M.K. Pavicevic, Fac. of Mining \& Geology, Univ. of Beograd, Dusina 7, POB 244, YU-11000 Beograd

20- 24

PM '91: Int. Conf. on Physics of Irradiation Effects in Metals

G. Szenes, Inst. for General Physics, Eötvös Univ. Museum krt. 6-8, POB 323, H-1445 Budapest

$20-24$ Dubna, USSR

1st. Int. Workshop on Materials Processing at High Gravity

L. Regel, W.R. Wilcox (a), M. Rodot (b) $-1++7$ (095) 3107023

(a) $-1++1$ (315) 2686438 ; (b) - I + +33 (1) 45344696 Smolence, Czechoslovakia

6th Symp. on Weak Superconductivity

S. Benačka, Slovak Academy of Sciences, CS-842 39 Bratislava

++42 (7) $3782139 / 3782470$

A: 31 Jan 91 / PP / $80 /$ SFR 400.-, incl. board, lodge, incl. proc.

$20-24$

Aarhus, Denmark

Strange Quark Matter

J. Marsden, Aarhus Univ., Denmark

Bitnet: jesm @ alliant.uni-c.dk

21 - 24

Adriatico Res. Conf.: Structural and Phase Stability of Alloys

Adriatico Res. Conf., ICTP, POB 586, 1-34100 Trieste ++39 (40) $224241 / 224531$

A: 15 Mar $91 / 70 /$ inv. / ICTPP SISSA

\section{1 - 24 May}

Brussels, Belgium

IPAT 91 : 8th Int. Conf. on Ion and Plasma Assisted Techniques

IPAT Secretariat, CEP Consultants Ltd. 26-28 Albany St., Edinburgh, EH1 30H, UK ++44 (31) $5572478 / 5575749$ Ab: 16 Nov $90 /$ PP: 15 Feb 91 BF 16800.-, incl. proc

$21-25$

Münster, Germany

10th Int. Conf. on Solid Compounds of Transition Elements

W. Jeitschko, Anorg.-Chem. Inst., Univ. Münster, W-4400 Münster

$21-25$

Heraklion, Crete, Greece ATO Adv. Res. Workshop: Proton Transfer in Hydgrogen-bonded Systems

S. Pnevmatkos, Univ. of Crete, Foundation for Research and Technology, POB 1527, GR-71110 Heraklion

$21-25$

Jahrestagung DGaO 1991

++49 (441) $7983454 / 7983201$

Ab: 11 Jan 91 / DM 100.-; 20.- students;

80. - members DPG and DGaO

21 - 25

Praha, Czechoslovakia

Centre for Theo. Study Workshop No. 1: Evolution of Interstellar Matter \& Dynamics of Galaxies

J. Palous, Astronomical Inst. Czechoslovak Acad. of Sciences, Budecska 6, CS-120 23 Praha 2

22 - 23

Belfort, France

Coll. Annuel de Thermique: Méthodes Expérimentales en Thermique

Sec. du Coll. de Thermique S.F.T. 91, Inst. de Génie Energétique, Parc Technologique 2 av. Jean Moulin, F-90000 Belfort

++33 () $84578200 /-$

Ab: 15 Jan 91/A: 1 Apr 91 / French

\section{Meetings Listings}

The Europhysics Meetings Database carries a complete listing of physics meetings worldwide of interest to European physicists. Listings sorted by date, venue and field are available as follows:

- Short Entry:

date, title and venue only

SFR 50. - per 50 entries

- Full Entry

complete database entry SFR 50. - per 20 entries

Orders: EPS Secretariat, Geneva. minimum order: SFR 50.- 
22 - 25 May

La Biodola, Isola d'Elba, Italy

4th Blois Workshop: 4th Int. Conf. on Elastic and Diffractive Scattering

F. Cervelli, INFN, Sezione di Pisa, via Livornese, 582/a, 1-56010 S. Piero a Grado (Pisa) ++39 (50) $546111 / 589047$ vaxpi @ 4bloispisa

\section{Ghent, Belgium}

Gen. Scientific Meeting of the Belgian Physical Soc

C. Henriet-Iserentant, Lab. voor Magnetisme, Rijksuniversiteit Ghent, Proeftuinstraat 86, B-9000 Ghen

++32 (91) $228731 / 227512$

A: 15 Apr 91 / Ab: 15 Apr 91 / PP /

- 29

Tucson, AZ, USA

4th Conf. on the Intersections Between Particle and Nuclear Physics

E.D. Zukowski, Building 510 F, Brookhaven Nat. Lab., Upton, NY 11973-5000, USA ++1 (516) $2823866 / 2825820$

26 - 30 Bitnet: henp @ bnldag

EAEG Ann. Assembly

Firenze, Italy

EAEG, POB 298, NL-3700 AG Zeist

26 - 30
5th Pisa Meeting on Advanced Detectors +31 (3404) $56997 / 62640$

INFN, Sezione di Pisa, via Livornese, 582/A

1-56010 S. Piero a Grado, Pisa

++39 (50) $546111 / 589047$

Bitnet: piameet @ ipivaxin

A: 30 Apr 91 / PP / 150 / inv. / SFR 1000.-,

incl. board, lodge, incl. proc. / INFN, SIF

\section{6 - 31 \\ Stockholm, Sweden}

Solidification Processes in Polymers: 23rd Europhysics Conf. on Macromolecular Physics

EPS-23, Stockholm Convention Bur. POB 6911, S-102 39 Stockholm ++46 (8) $230990 / 348441$ Ab: 31 Mar 91 / PP / SKR 2800.2200. - IOM's; 800. - students (2600, - : 3300 1000. - after $15 \mathrm{Mar} 91)$

27 - 31 Kazimierz, Poland

Puzzles on the Electroweak Scale: XIV Int. Warsaw Meeting on Elementary Particle Physics

Z. Ajduk, Inst. of Theo. Physics, Warsaw Univ., Hoza 69, PL-00-681 Warsaw $-I++48$ (22) 219475

825548

A: 31 Mar 91 / Ab: 15 Apr 91 / PP: 30 Sep 91 $100 /$ \$US 150.-, incl. board, lodge

27- $31 \quad K e r k r a d e$, The Netherlands

IUTAM Symp. on Numerical Solution of the Nonisothermal Flow of Viscoelastic Liquids

J.F. Dijksman, Philips Res. Labs., POB 8000 , NL-5600 JA Eindhoven inv. / IUTAM

$27-31$

Madrid, Spain

Radionucleide Metrology and its Applications

H.M. Los Arcos, ICRM'91 Secretariat, CIEMAT/Investigacion Bàsica,

Avenida Complutense, 22, E-28040 Madrid

++34 (1) $3466225 / 3466005$

EARN: arcos @ emdjenn11

A: 1 Apr 90/Ab: 1 Dec 90/PP: 15 Apr 91/100/ PTAS 7000.-, incl. proc.

27 - 31

Strasbourg, France

European Materials Res. Soc. Spring Meeting \& ICAM-91: Int. Conf. on Adv. Materials

P. Siffert, E-MRS, C.T.T., BP 20 ,

F-67037 Strasbourg Cédex

++33 () $88286543 / 88280920$

A: 1 Apr 91 / Ab: 15 Mar 91 /FF 2000.- ; 900. - students (2200. - :

1100. - after 15 May 91), incl. proc. / IUMRS

27 May-1 June Brioni Islands, Yugoslavia Heavy lon Physics - Today and Tomorrow: 7 th Adriatic Int. Conf. on Nuclear Physics

R. Caplar, Adriatic Conferences, Rudjer Boskovic Inst. Bijenicka 54, POB 1016, YU-41 001 Zagreb ++38 (41) 435111 ext. $366 / 425497$

A: 30 Mar $91 / \mathrm{Ab}: 15 \mathrm{Apr} 91 / \mathrm{PP}$

$28-30$

Maastricht, The Netherlands

12th SAMPE Int. European Conf.

M. Smith, SAMPE, POB 2459, Covina, CA 91722, USA $++1(818) 3310616 /$

28 May-1 June Copenhagen, Denmark

NATO Adv. Res. Workshop: Quantum Chaos - Theory and Measurement

E. Pedersen, NORDITA, Blegdamsveg 17

DK-2100 Copenhagen

++45 (31) $421616 / 389157$

epedersen@ nbivax.nbi.dk

A: 1 Feb 91 / PP / 150 / DKR 500.- ; students free

\section{9 - 31 May \\ Sant Feliu de Guixols, \\ Gerona, Spain}

6th European Workshop on Molecular Spectroscopy and Photon-Induced Dynamics

A. Aguilar, Dept. de Quimica Fisica Fac. de Quimica, C/ Marti i Franques 1 E-08028 Barcelone ++34 (3) 3307311 ext. 1207 / 4111492

d3qfaan0@ ebOub011

A: 15 Mar 91 / Ab: 15 Mar $91 /$ PP: 29 May 91 / 80 / PTAS 17000.15000. - IOM's; 10000. - students, incl. proc. / DGICYT, CE

29 - 31 Porto Carras, near Thessalonika, Greece NATO Adv. Res. Workshop: Status and Future Developments in the Study of Transport Properties

W.A. Wakeham, Dept. of Chemical Engng. and Chem. Technology, Imperial Coll. of Sci. and Tech., Prince Consort Rd., London, SW7 2BY, UK

29- 31 Rotterdam, The Netherlands 8th European Microelectronics Conf.

Eurocongress, Amstel 268, NL-1017 AM Amsterdam

30 May - 2 June

Int. Optometric and Optical League: Gen. Delegate Meeting

Int. Optometric \& Optical League

10 Knaresborough Place, London, SW5 OTG, UK

++44 (71) $3704765 / 3731143$ $\mathrm{NP} / 70$

30 May - 4 June

BT-91: 3rd Int. Conf. on Electron Beam Technologies '91

EBT-91 Secretariat, Inst. of Electronics,

72 Lenin Bd., BG-1784 Sofia

++359 (2) 74311 ext. 582 / -

23561

PP: 20 Dec 90 / Russian / \$US 200.-

June

Cardiff, UK

9th Conf. on Heat Transfer of the Unione Italiana de Thermfluidodinamica

S. Faggiana, Dipt. di Energetica, Fac. di Ingegneria via Diotisalvi, 2, 1-56126 Pisa

Ab: 15 Jan 91 / A: 25 Apr 91 / Italian

2- 5 Orlando, FL, USA

American Nuclear Soc. Ann. Meeting

American Nuclear Society, 555N. Kensington Ave. La Grange Park, IL 60525, USA ++1 (708) $3526611 / 3526464$

3- 5 Copenhagen, Denmark

NORDITA Symp.: Physics of Quantum Chaos and Measurement

E. Pedersen, NORDITA, Blegdamsveg 17

DK-2100 Copenhagen

++45(31) $421616 / 389157$

epedersen@ nbivax.nbi.dk

A: 1 Feb 91 / NP / 100 / DKR 500. - ; students free

3-6 Espoo, Finland

IGARSS '91: Remote Sensing - Global Monitoring for Earth Management

M. Hallikainen, Helsinki Univ. of Tech., SF-02150 Espoo ++356 (0) $4512264 / 460224$

\section{3-7 Berlin, Germany \\ 18th Conf. on Controlled Fusion and \\ Plasma Physics \\ M. Blaschke, ZIE der AdW, \\ Hausvogteiplatz 5-7, РОВ 1250 , \\ O-1086 Berlin \\ ++37 (30) $2612035 / 2612035$ \\ A: 31 Mar 91 / Ab: 11 Jan 91 / \\ PP: 31 Mar 91 / DM 550.-: \\ 450. - IOM's; 75. - students}

3-7

Heidelberg Germany

Conf. on Physics of Active Galactic Nucle

Landessternwarte Königstuhl, AGN Konferenz, W-6900 Heidelberg

3- 7 $\mathrm{PP} /$ limited to 160

Int. Conf, on Nuclear and Atomic Clusters

CLUSTERS '91, Dept. of Physics, Abo Akademi

Porthansgatan 3, SF-20500 Turku

++356 (21) $654246 / 654776$

cluster@ finabo

Ab: 15 Mar 91 / PP / inv.

3- 7

Ustron-Jaszowiec, Poland

4th Int. Symp.: Physics of Metals

E. Talik, Inst. of Physics, Univ. of Silesia,

Uniwersytecka 4, PL-40-007 Katowice

A: 1 Mar 91 / Ab: 1 Apr 91 / PP /

\$US 200,- incl, board, lodge

3-8 June San Feliu de Guixols, Spain

20th G.I.F.T. Int. Seminar on Theoretical Physics: Quantum Gravity and Cosmology

J. Pérez Mercader, C.S.I.C., Madrid

++34 (1) $4112962 /$ -

mercader@goya.dit.upm.es

A: 31 Mar 91 / PP

$3-29$

Beg-Rohu, France

Hydrodynamique et Turbulence

C. Godrèche, SPSRM, CEA Saclay,

F-91191 Gif-sur-Yvette Cédex

++33 (1) $69087263 / 69088786$

Bitnet: godrech @ frsac11 A: 15 Feb 91 / FF 5000

7th Interdisc. Workshop: Nonlinear Coherent Structures in Physics

M. Peyrard, Lab. Ondes et Structures Cohérentes, Univ. de Bourgogne, 6, bd. Gabriel, F-21100 Dijon ++33 () $80396043 /-$ peyrard/remsnet @ frccub11

A: 15 Apr 91 / limited to 60 / FF 300.-

5-7 Nijmegen, The Netherlands

European Conf. on Metallorganic Phase Epitaxy

Univ. Congress Bureau, POB 9111, NL-6500 Nijmegen $\mathrm{HN}$

$10-14$

Dubrovnik, Yugoslavia

-5: 5th Int. Symp. on Radiation Physics

A. Ljubicic, Ruder Boskovic Inst., ISRP-5,

POB 1016, YU-4101 Zagreb

++38 (41) $425653 /$ -

A: $15 \mathrm{Apr} 91$ / Ab: 14 Apr 91 / PP / 250 / \$US 220.-: 100. - students, incl. proc.

$10-14$

Kanazawa, Japan

4th Int. Conf. on Nucleus-Nucleus Collisions

1. Tanihata, RIKEN, 2-1 Hirosawa, Wako,

Saitama 351-01, Japan

$-1++81$ (484) $624643 /$

PP: 28 Feb 91 / IUPAP

$10-14$

Antibes, France

CIP 91: 8th Int. Coll. on Plasma Processes

J. Fauvet, SfV, 19, rue du Renard, F-75004 Paris

++33 (1) $42781582 / 42786320$

Ab: 21 Jan 91 / PP: 2 Apr 91 / French /

FF 3700. - ; 3950. - after 2 May 91, incl. proc

$10-14$ München, Germany

Laser '91: Optoelectronics and Microwaves

MMG-Munich

10 - 14

San Jose, CA, USA

Physics Computing ' 91 (incl. 3rd Int. Conf. on Computational Physics)

M. Mays, Lawerence Livermore Nat. Lab. POB 5509 L-560, Livermore, CA 94550, USA ++1 (415) $4224001 /$ Ab: 1 Feb $91 /$ PP / APS, AIP

10- 21 Cetraro, Italy

v. Study Inst.: Metal-Ligand Interactions: From Atoms, to Cluster, to Surfaces

Salahub, Dept. of Chem., Univ. of Montreal, CP6128, Succ. A, Montreal, Quebec, $\mathrm{H} 3 \mathrm{C} 3 \mathrm{~J} 7$, Canada

11 - 14 Trieste, Italy

Adriatico Res. Conf.: Physics of Inhomogeneous Materials

Adriatico Res. Conf., ICTP, POB 586, 1-34100 Trieste ++39 (40) $224241 / 224531$ A: 15 Mar $91 / 70 /$ inv. / ICTP, SISSA

$11-14$

Int. Conf.: Physics for Industry Malinowski, Inst. of Applied Sciences, Moldavian SSR, Grosul stz 5, SU-277028 Kishinev-28 $++7(422) 725887 /$ -

Ab: 28 Feb 91 / PP / \$US 100.-, incl. proc. / IUPAP

12-15 Berlin, Germany

Dynamic Days Berlin: 12th Annual Workshop

G. Eilenberger, IFF, KFA Jülich, Postfach 1913 , W-5170 Jülich 
17 - 21 June

Smolenice, USSR

Wh Workshop on Dynamical Aspects of Nuclear Fission

J. Kristiak, Inst. of Physics, Slovak Acad. of Sciences ad JNIR Dubna, Dubravaska Cesta 9, Bratislava

17 - 21

Columbus, OH, USA

46th Symp. on Molecular Spectroscopy

Ohio State Univ., Dept. of Physics, 174 W 18th Ave. Columbus, $\mathrm{OH} 43210$, USA

$17-21$

3rd Topical Seminar on Heavy Flavours

P.G. Pelfer, Dip. di Fisica, Univ, di Firenze,

Largo E. Fermi, 2, 1-50125 Firenze

++39 (55) $2298141 / 229330$

flavour91 @ fi.infn.it

A: 31 Mar $91 / 100$

17 - 21

Como, Italy

European Research Conf, in Physics: Quantum Groups and Topology in Field Theory and Superconductivity

J. Hendeković, ERC Programme, ESF,

1, quai Lezay Marnésia,

F-67000 Srasbourg

++33 () $88255747 / 88366987$

17- 21 Font-Romeu, France

TENICOLS '91:10th Int. Conf. on Laser Spectroscopy

M. Ducloy, Univ. de Paris-Nord, Lab. de Physique

des Lasers, F-93430 Villetaneuse

++33 (1) $49403400 / 49403200$

A: 1 Dec 90 / Ab: 15 Dec 90 / PP / limited to 250 / inv. / FF 1500. - / IUPAP

18 - 21

Pittsburgh, PA, USA

5 th Joint Magnetism and Magnetic Materials (MMM) and Int. Magnetics (Intermag) Conf.

D.Suiters, Courtesy Associates, Inc., 655 15th St., NW Suite 300, Washington D.C. 20005, USA ++1 (202) $6395088 / 3476109$ Ab: 23 Jan 91 / PP: 15 Apr 91 / \$US 255.-

18 - 21 Darmstadt, Germany

MMW-7: 7th Int. Magnetic Measurement Workshop

S. Raiss, GSI, Postfach 110 552, W-6100 Darmstadt ++49 (6151) $359412 / 359785$ Bitnet: ul22@ddgags13

20 - 22

Colmar, France

11th Int. Conf. on Physics in Collision

Huss, Univ, de Haute Alsace, 61, rue Albert Camus, F-68093 Mulhouse Cédex

++33 (89) $424846 / 434555$ physcoll @ frccsc21

$23-28$

Aussois, France

Coll. Weyl VII: Int. Conf. on Metals in Solution

Leclercq, Lab. de Chimie Physique,

URA 253 du CNRS-HEI, 13, rue de Toul,

F-59046 Lille Cédex

++33 () 20308314 ext. $547 / 20428146$

chiphy2@ frfupl11

A: 15 Feb 91 / Ab: 15 Mar 91 / PP / FF 1000.EHI, CNRS, ILL

$23-29$

Irsee, Germany

NATO Adv. Res. Workshop: Ordered Intermetallics Physical Metallurgy and Mechanical Behaviour

C.T. Liu, Oak Ridge Nat. Lab., POB 2008, Oak Ridge, TN 37831-6115

$23-29$

Kyoto, Japan

6th Marcel Grossmann Meeting on General Relativity

MG6 Secretariat, Dept. of Physics, Kyoto Univ., Kyoto 606, Japan

++81 (75) $7533844 / 7119379$

Bitnet: mg6 @ jimrifp

A: 30 Nov $90 / \mathrm{Ab}: 28$ Feb 91 / PP / 550 /

Y $30000 .-$; $35000 .-$ after 1 Apr 91, incl. proc. IUPAP, PSJ

$23-29$

lerapetra, Crete, Germany

Int. Conf. on New Nuclear Physics with Adv. Techniques

S. Kossionides, Inst. of Nuclear Physics,

NRCPS Demokritos, GR-153 10 Agia Paraskevi

++30 (1) $6518770 / 6511215$

Bitnet: tandem @ grathdem

A: 31 Jan 91 / Ab: 1 Apr 91 / SFR 260.-

$24-28$

Int. Conf. on Artifical Neural Networks

Espoo, Finland

O. Simula, Helsinki Univ. of Tech., SF-02150 Espoo

24- 28 Weimar, Germany

Radiation Effects in Insulators - 6

G. Götz, Friedrich-Schiller-Univ. Jena,

Max-Wien-Platz 1, 0-6900 Jena

++37 (78) 82254141 -

588634

A: 1 Oct 90 / PP / 200 / \$US 350. 200. - students, incl. proc.

24- 29

Jülich, Germany

7 th Int. Conf. on Real Time Computer Applications in Nuclear, Particle and Plasma Physics

K.D. Muller, KFA Jülich, Postfach 1913, W-5170 Jülich ++49 (2461) $614666 / 614666$ wta101@ djukfa11
25 - 27 June

Glasgow, UK

ISEC '91: Int. Superconductive Electronics Conf.

Conf. Secretariat, ISEC '91, Meeting Makers, 50 Richmond St., Glasgow, G1 1XP, UK

++44 (41) $5531930 / 55320511$

Ab: 25 Feb 91 / PP / f 190.

(215. - after 14 May 91); 100. - students

Trondheim, Norway

Norwegian Phys. Soc. Ann. Meeting

A. Reitan, Dept. of Physics, AVH/UNIT,

N-7055 Dragvoll

++47 (7) $591864 / 591852$

A: 3 May 91 / Ab: 3 May $91 / N P$ / 150 /

Norwegian / NKR 500 .

$25-28$

Bad Honnef, Germany

Int. Conf. on Surface X-ray and Neutron Scattering

H. Zabel, Fak. f. Physik und Astronomie, Ruhr-Univ.

Bochum, Postfach 1021 48, W-4630 Bochum 1

++49 (234) $7003649 / 7002001$

$25-28$

Nice, France

NATO Adv, Res. Workshop: Lattice Gas Automata

J.P. Boon, Univ, Libre de Bruxelles, Fac. des Sciences, Campus Plaine, CP231, B-1050 Bruxelles

$25-29$

4th Int. Symp. on Heavy Flavour Physics

N. Mathieu, Lab. de I'Accélérateur Linéaire,

Univ. de Paris-Sud, Bât. 200 ,

F-91405 Orsay Cédex

++33 (1) $64468437 / 69079404$

flavour@ frcpn11

inv/ \$ US 120,-

July

18th Ann. Plasma Summer Conf.

Brighton, UK

T.N. Todd, Cullingham Lab., Abingdon,

1 - 4

xford, OX 14 3DB, UK

Ultrasonics International '91

M. Vukovojac, Butterworth Scientific Ltd

POB 63, Westbury House, Bury St., Guildford,

Surrey, GU2 5BH, UK

++44 (483) $300966 / 301563$

1 - 5 b: 2 Nov $90 / P P$

IBA-10: 10th Int. Conf on Ion Beam Analysis

Congress Office, Eindhoven Univ. of Technology,

POB 513, NL-5600 MB Eindhoven

$++31(40) 474000 / 455925$

PP / FLR 550. - ; 400. - students, incl. proc.

$1-5$ Torino, Italy

Nonlinear Stochastic Mechanics

N. Bellomo, Dip. di Mathematica, Politecnio di Torino, Corso Duca degli Abruzzi 24, I-10129 Torino

$7^{\text {inv }}$

Bath, UK

Int. Conf. on the Mathematics of Nonlinear System

J.F. Toland, School of Math. Sciences, Univ. of Bath

Claverton Down, Bath, BA2 7AY, UK

7th IAP Astrophysics Meeting: Extragalactic Radio

Sources - From Beams to Jets

2-6 Trieste, Italy and Neural Networks

Adriatico Summer Prog. on Complex Systems, ICTP, POB 586, 1-34100 Trieste

3- 7 +39 (40) $224241 / 22453$

Erice, Italy Physics at the Highest Energy and Luminesity

A. Zichichi, EP Div., CERN, CH-1211 Geneva 23 NATO

Int. Conf. on Atomic and Molecular Dynamics

C.T. Chang, Inst. of Atomic and Molecular Sciences, Academica Sinica, $\mathrm{POB}$

7 - 11 $+886(2) 3620200$

8th COMPUMAG Conf. on the Computation of Electromagnetic Fields

COMPUMAG Secretariat, Dipartimento di Ingegneria Elettrica, Universita di Napoli "Federico II", via Claudio, 21, 1-80125 Napoli

++39 (81) $7683250 / 616897$

uncompum @inacriai.earn

Ab: 15 Nov 90 / A: 8 Jul $91 / 250$

sUS 250.-, incl, proc.

8 - 12

Cambridge, UK

IWCC-6: 6th Int. Workshop on Critical Currents

Conf. Organizer, Cambridge Programme, Dept. of Engng, Trumpington St., Cambridge, CB2 1PZ, UK - 12 b: 14 Jun 91 / PP: 8 Jul 91 / limited to 150

Dijon, France

EMF7: 7th European Meeting on Ferroelectricity

annot, EMF-7 Secretariat, Lab. de Physique du

Solide, BP 138, F-21004 Dijon Cédex

++33 () $80395947 / 80395069$

jannot @ frccub11

Ab: 31 Dec 90 / PP: 8 Jul 91 / FF 1800. - ; 1000. -

students (2000. - ; 1200. - after 30 Apr 91)

8- 11 July

Moscow, USSR

NAN-91: Workshop on Nucleon Antinucleon Interactions

N. Smorodinskaya, Inst. of Theor. and

Experimental Physics,

B. Cheremushkinskaya 25, SU-117259 Moscow

8- 12 Barga (LU), Italy lonized Gases

V. Palleschi, ICPIG XX, Ist. di Fisica Atomica e Molecolare-CNR, via del Giardino, 7, 1-56127 Pisa ++39 (50) $543456 / 589048$ ifamdue@ icnucevm.cnuce.cnr.it PP: 31 Jan 91 / 100 / LIT 300000.- ; 225000.- students (400000. 300000.- after 1 May 91), incl. proc. / IUPAP, CNR. SIF

8 - 12

3rd. Int. Conf. on Chemical Analysis:

Namur, Belgium Microanalysis of Light Elements Using Charged Particles Accelerators

Ch. Honhon, L.A.R.N., rue Muzet 22, B-5000 Namur ++32 (81) $731267 / 230391$

8 - 9 Phase Flows

M. Alaphilippe, Inst. Universitaire de Technologie,

Dépt. Génie Thermique et Energie,

av. de I'Université, F-64000 Pau

++33 () $59923374 /-$

A: 1 Jan 91 / ECU 250.-, incl. proc

9- 11

Grenoble, France Medecine and Biology, Physics and Chemistry

F. Stoeckel, Lab. de Spectrométrie Physique, BP 87, Univ. Joseph Fourier - Grenoble,

F-38402 Saint Martin d'Hères Cédex

$++33076514749 / 76514545$

Ab: 15 Mar 91 / NP / 350 / French, German / CNRS

$9-124$

23rd EGAS Conference

Torun, Poland

S. Legowski, Inst. of Physic

Nicolas Copernicus Univ.

ul Grudziadzka 5/7, PL-87-100 Torun

$-1++48$ (56) 24436

A: 11 Apr 91 / Ab: 20 May 91 / PP / $200 /$

\$US 100.-, incl. proc.

9- 12

Trieste, Italy

Adriatico Res. Conf.: Open Problems in Strongly Interacting Electron Systems

Adriatico Res. Conf., ICTP, POB 586, 1-34100 Trieste ++39 (40) $224241 / 224531$

A: 15 May $91 / 70$ / inv. / ICTP, SISSA

10- 16 Brisbane, Australia

ICPEAC 1991: 17th Cont. on the Physics of Electronic and Atomic Collisions

Secretary, XVII ICPEAC Conf., GPO Box 853, Brisbane, Queensland 4001, Australia

++61 (617) $2212762 / 2200231$

A: 15 Apr 91 / Ab: 15 Feb 91 / PP / 600

SUS 300 - : 190 - students, incl, proc. / IUPAP

$15-17$

Meudon-Bellevue, France

2nd Conf, on Beam Injection Assessment of Defects in Semiconductors

B. Sieber, Lab. de Structure et Propriétés de

'Etat Solide, URA 234, Bât. C6,

Univ. des Sciences et Techniques de Lille,

F-59655 Villeneuve d'Ascq Cédex $++3320434913 / 20436591$

$15-19$ 


\section{7 - 19 July} SfV, 19, rue du Renard, F-75004 Paris 18 - 19

Marseilles, France +33 (1) $42781582 / 42786320$ (n) High Energy Physics Int. Centre for Theo. Physics, POB 586, I-34100 Trieste ++ (40) $22401 / 224163$

22 - 24

Cambridge, UK

Euromat '91: 2nd European Conf. on Advanced Materials \& Processes

Euromat '91, IOM, 1 Carlton House Terr. London, SW1Y 5BD, UK ++44 (71) $8394071 / 8393576$

$22-26$ Dublin, Ireland

IMACS '91: 13th IMACS World Congress on Computation and Applied Mathematics

P. McKeever, IMACS '91 Secretariat, 26 Temple Lane, Dublin 2, Ireland

++353 (1) $452081 / 451739$

jmiller@ vaxi.tcd.ie

A: 15 Mar 91 / Ab: 15 Mar 91 / PP: 15 Mar 91 \$IRL 255. - ; (285. - after 15 May 91); 25. - students, incl. proc

22- 26 Nijmegen, The Netherlands European Research Conf.: Molecular Energy Transfer J. Hendeković, ERC Programme, ESF. 1, quai Lezay Marnésia, F-67000 Srasbourg ++33 () $88255747 / 88366987$ A: $22 \mathrm{Apr} 91 / \mathrm{NP} / 80 / \mathrm{CEC}$, ESF

$22-26$

Kanazawa, Japan

Int. Conf on Materials and Mechanisms of Superconductivity - High Temperature Superconductors

K. Kitazawa, Dept. of Ind. Chemistry, Univ. of Tokyo Hongo, Bunkgo-ku, Tokyo 113, Japan $\mathrm{Ab}: 31$ Mar 91

23 July - 1 Aug.

Buenos Aires, Argentina

Int. Astronomical Union: 21st Gen. Ass.

Local Org. Committee, Sarimiento 1562, 4F,

1042 Buenos Aires, Argentina

A: 10 May 91 / \$US 150.-, 200.- after 10 May 91

\section{July - 1 Aug. Geneva, Switzerland}

LP-HEP 91 : Joint Lepton-Photon Symp. and Int. Europhysics Conf. on High Energy Physics

L. Griffiths, LP-HEP 91 Conf. Secretariat, CERN, $\mathrm{CH}-1211$ Geneva ++41 (22) $7672755 / 7672100$ Iphep91@ cernvm.cern.ch Ab: 3 May 91 / PP: 14 Jun 91 / 1000 / inv. SFR 300.- ; (350.- after 1 Jun 91): 270. - IOM's; students free I IUPAP, CERN

28 July - 2 Aug

Salford, UK

ICACIS-14: 14th Int. Conf. on Atomic Collisions in Solids

S. Lippmann, The Institute of Physics

47 Belgrave Sq., London, SW1X $80 \times$, UK

++44 (71) $2356111 / 2596002$

A: 30 Jul 91 / Ab: 28Feb 91/PP: 28 Apr 91/200/ incl. proc.

28 July - 2 Aug.

ICP-XV: 15th Int. Conf. on Photochemistry

E. Migirdicyan, Lab. de Photochimie Solaire, 2-8 rue H. Dunant, F-94320 Thiais

30 July - 2 Aug.

Vacuum' 91

S. Lippmann, The Institute of Physics, 47 Belgrave Sq., London, SW1X 80X, UK ++ (71) $2356111 / 2596002$ A: 28 Jun 91 / Ab: 22 Mar 91 / PP: 30 Jul 91 / A: 80 -, incl. proc.

30 July - 8 Aug.

IAMP Cong. on Mathematical Physic Karl-Marzs. F. Theoretische Physik, (10.70-7010 Leipzig PP / DM 240.- ; 300. - after 1 Jun 91, incl. proc.
UUPAP Aug.

IAGA Session: Historical Data and the Variability of Solar and Geomagnetic Activity

Geophysikalische Station, Hechelstr. 8 W-2820 Bremen-Roennebeck

2-6 Buenos Aires, Argentina

IAU Coll. No. 133: Eruptive Solar Flares

Z. Svestka, SRON Lab. for Space Res.,

Sorbonnelaan 2, NL-3584 Utrecht

3-7 Campos de Jordao, Brazil

AU Symp. No. 150: The Astrochemistry of Cosmic Phenomena

Harvard-Smithsonian Center for Astrophysics, 60 Garden St., Cambridge, 02138 MA, USA

5 - 8 Cordoba, Argentina
IAU Symp. No. 151: Evolutionary Processes in Interacting Binary Stars

Y. Kondo, Code 684, Goddard Space Flight Center, Greenbelt, MD 20771, USA
5 - 9 Aug.

Angra dos Reis, Brazil

IAU Symp No. 149: The Stellar Populations of Galaxies A. Renzini, Dipt. d'Astronomia, CP 596. 1-40100 Bologna

38th Int. Field Emission Symp.

Wien, Austria

. Mitterauer, Inst. f. Alleg. Elektrotechnik u. Elektronik, Tech. Univ. Wien, Gusshausstr. 27-29,

A-1040 Wien

$++43(222) 588013870 / 5052666$

\section{Amsterdam, The Netherlands \\ 1991 EPS Nuclear Physics Conf. on Hadronic Structure and \\ Electromagnetic Interactions \\ J.H. Koch, NIKHEF, POB 41882 \\ NL-1009 DB Amsterdam \\ ++31 (20) $5922163 / 5922165$ \\ epscon@ nikhefk.nikhef.nl \\ A: 15 Mar 91 / Ab: 1 May 91 / PP / 300 / \\ FLR 550-: 450- IOM's: 500. - Nat. Socs., incl. proc.}

6 - 9 Middlefart, Denmark

STOP 91: Int. Conf. on Stopping of Low- and High- Z lons

P. Sigmund, Physics Dept., Odense Univ.

DK-5230 Odense $M$

++45 (66) 158600 ext. $3522 / 158760$ psi@ dou.dk

A: 1 May 91

Workshop on Superstrings and Related Topics

Int. Centre for Theo. Physics, POB 586, 1-34100 Trieste $++(40) 22401 / 224163$

$11-13$

22nd Int. Conf. Cosmic Ray Conf.

Dublin, Ireland

D. O'Sullivan, Dublin Inst, for Advanced Studies,

5 Merrion Sq., Dublin 2, Ireland

++353 (1) $774321 / 682003$

Bitnet: icrc@irlearn

A: 1 Feb 91 / Ab: 1 May 91 / PP: 23 Aug 91/550/ fIRL 250 . -, incl. proc. / IUPAP, DIAS

$11-24$

Vien, Austria

20th Gen. Ass. of the Int. Union of Geodesy and Geophysics

P. Steinhauser, ZAMG, Hohe Warte 38, A-1191 Wien ++43 (1) $222364452-2001 / 2223691233$ French

12 - 16

Interlaken, Switzerland

STM '91: 6th Int. Conf. on Scanning Tunneling Microscopy

Ch. Gerber, IBM Research Div., Zürich Res. Lab., $\mathrm{CH}-8803$ Ruschlikon

++41 (1) $7248645 / 7243223$

Ab: 15 Apr 91 / PP: 12 Aug 91 / SFR 550. - (550. after 10 Jun 91); 200.- students, incl. proc. / IUC

E 17-20 Skofja Loka (Slovenia), Yugoslavia

Europhysics Study Conf.: The Role of Experiment in Physics Education

S. Oblak, Zavod RS Solstovo, Poljanska 28

YU-61000 Ljubljana

++38 (61) $319066 /$ -

$70 /$ \$US 150.-; 100.- students

$17-21$

Brussels, Belgium

11 th Quadrennial Cong. of the Int. Comm. on Rheology

Unité de Mécanique, Univ. Catholique de Louvain Bât. Simon Stevin , 2, place du Levant. B-1348 Louvain-la-Neuve

\section{8 - 21 \\ Davos, Switzerland \\ Europhysics Industrial Workshop: EIW-8} Nanometer-Scale Methods in Technology \& Industry

J.K. Gimzewski, IBM Research Div., Forschungslab. Zürich, Säumerstr. 4 $\mathrm{CH}-8803$ Rüschlikon

A: 30 Apr 91 / Ab: 30 Apr 91 / NP /

limited to 50 / SFR 600 -.

400.- AM's \& IOM's, incl. board, lodge

19 - $21 \quad$ Louvain-la-Neuve, Belgium

2nd Int. Conf. on Radioactive Nuclear Beams

J. Vervier, Inst. de Physique Nucléaire,

ch. du Cyclotron 2, B-1348 Louvain-la-Neuve IUPAP

19- 22

PF'91: Particles and Fields ' 91

TRIUMF, 4004 Westbrook Mall,

Vancouver, BC, V6T 2A3, Canada

++1 (604) $2221047 /$ -

Bitnet: pf91 @ triumfcl

APS, Can. Ass. Physicists

19- 23 Garmisch-Partenkirchen, Germany

CAS 14: 14th Int. Conf. on Amorphous Semiconductors - Science and Technology

W. Fuhs, Fachbereich Physik, Philipps Univ. Marburg Renthof 5, W-3550 Marburg

++49 (6421) $284246 / 284239$

Bitnet: fuhs@ dmrhrz11

Ab: 15 Mar 91 / PP: 31 Jul 91 / IUPAP

19 - 24 Aug.

Torun, Poland

IIREP '91 Int. Conf.: Teaching about Reference Frames - From Copernicus to Einstein

J. Turlo, Instytut Fizyki, Copernicus Univ., PL-87 100 Torun

++48 (56) $29216 / 24602$

A: 1 Jun 91 / Ab: 15 Mar 91 / PP / $300 /$

\$US 120.-; 100.- IOM's; $50 .-$ students / IUPAP, UNESCO

20- 24

Balatonszeplak, Hungary

MOSPOQ '91: Int. Conf. on Polytype Modulated Structures and Quasicrystals

MOSPOQ '91 Secretary, Roland Eötövos Phys. Soc., POB 433, H-1371 Budapest

20 - 27

20 - 27
6th Workshop on Hadronic Mechanics Marino, Italy Palm Harbour, FL 34682-1577, USA - I + + (813) 9349593 9349275

21 - 23

Stockholm, Sweden

Conf.on liquid Noble Gas Detectors and their Applications

T. Lindblad, Manne Siegbahn Inst. of Physics, S-10405 Stockholm

++46(8) $161109 / 158674$ lindblad@vand.physto.se

$25-30$ Zagreb, Yugosla
CMOS-20: European Congress on Molecular Spectroscopy

M. Meic, Ruder Boskovic Inst., PON 1016, YU-41001 Zagreb Ab: 15 Feb 91 / \$US 250.-

26- 29

Marburg, Germany (Satellite to ICAS 14)

P. Thomas, Fachbereich Physik, Univ. Marburg, Renthof 5, W-3550 Marburg

++49 (6421) $284219 / 284239$ Bitnet: thomasıp@dmrhz11

26 - 29 Dooorweth, The Netherlands

7 th Topical Int. Meeting on Photoacoustic Photothermal and Related Phenomen

D. Bicanic, Photoacoustic Laser Lab., Agricultural Univ., Postbus 9101, NL-6700 HB Wageningen

$++31(8370) 89111 /$ -

45917

Ab: 25 May 91 / PP / FLR 425. -

485. - after 15 Jun 91

26-30 Amsterdam, The Netheriands

IMSC-12: 12th Int. Mass Spectrometry Conf.

L. Roos, FOM, Kruislaan 407, NL-1098 SJ Amsterdam $++31(20) 946711 / 6684106$

Ab: 15 Jan 91 / PP / 1500 / FLR 500.550. - after 30 Jun 91 , incl. proc.

26 - 30

Coventry, UK Series from Mechanical Systems

G.P. King, Nonlinear Systems Lab., Mathematics Inst. Univ. of Warwick, Coventry, CV4 7AL, UK ++44 (203) $523698 /-$ IUTAM

26 - 30

ICMFS-91: 13th Glasgow, UK Surfaces

S. Lippmann, The Institute of Physics,

47 Belgrave Sq., London, SW1X $80 X$, UK

++44 (71) $2356111 / 2596002$

A: 26 Jul 91 / Ab: 3 Apr 91 / P: 15 Oct $91 / 200$ / f. 200.- approx. / IOP

26 - 30

Lausanne, Switzerland

16th Int. Conf. on Infrared \& Millimetre Waves

M.Q. Tran, CRPP, 21, av, des Bains, $\mathrm{CH}-1007$ Lausanne ++41 (21) $6933482 / 6935176$ immmw91 @ crppsun.epfl.ch 
27 - 30 Aug.

Oxford, UK

INCS '91: Int. Conf. on Neutron Scattering

M.B. Sherwen, ICNS '91 Secretariat, RutherfordAppleton Lab., Didcot, Oxon, OX11 OQZ, UK

++44 (235) $445553 / 445720$

icns @ uk.ac.rl.de

Ab: 15 Mar 91 / PP / f 185.- :

215. - after 15 Jul 91, incl. proc.

\section{$27-30$ \\ Edinburgh, UK \\ EOEC 91: 3rd European Quantum \\ Electronics Conf. and 10th Nat. \\ Quantum Electronics Conf. \\ Conf. Secretary, EQEC, Physics Dept. \\ Heriot-Watt Univ., Riccarton, \\ Edinburgh, EH14 4AS, UK \\ ++44 (31) $4495111 / 4513136$ \\ Ab: 1 Feb 91 / f 340.-; 270.- mbrs: \\ 195. - students (365.-; 295.-; 220. \\ after 20 Jun 91 ), incl. board, lodge / IOP}

$27-30$

Firenze, Italy

ICO Topical Meeting: Atmospheric, Volume and

Surface Scattering and Propagation

OIC/ICO Topical, via G. Moderna, 19, I-50121 Firenze $++39(55) 5000631 / 570227$

A: 31 Jan $91 /$ PP: 31 Jan $91 / 200$ /

LIT 300000.-; 95000.- students (340000.120000--after 1 May 91) / OSA, CNR

27 - $30 \quad$ Leiden, The Netherlands

DPC ' 91 : 8 th Int. Conf. on Dynamical Processes in Excited States in Solids

S. Völker, Huygens Lab., Rijksuniversiteit te Leiden, Postbus 9504, NL-2300 RA Leiden ++31 (71) $275700 / 275819$ $\mathrm{PP} / 200$

29 - 31

Amsterdam, The Netherlands

3rd. Int. Symp. on Research in High Magnetic Fields

P.H. Frings, Natuurkunde Lab., Univ. van Amsterdam, NL-1018X Amsterdam

Autumn

undecided

2nd Int. Workshop on Software Engineering, Artificia Intelligence and Expert Systems for High Energy and Nuclear Physics

D. Perret-Gallix, CERN EP Division, $\mathrm{CH}-1211$ Geneva 23 ++41 (22) $7672277 / 7824439$

Sept.

Padua, Italy

Workshop on Biophysical Modelling

K. Chadwick, CEC, DG XII/D/3, 200, rue de la Loi B-1049 Bruxelles ++32 (2) $2354334 /-$

1 - 5 Klingenthal, Germany European Research Conf.: Colloids and Interfaces J. Hendeković, ERC Programme, ESF, 1, quai Lezay Marnésia, F-67000 Srasbourg ++33 () $88255747 / 88366987$ limited to 100 / FF 2600.

$1-5$

Oxford, UK

ICCBE-3: 3rd Int. Conf. on Chemical Beam Epitaxy \& Related Growth Techniques

E.G. Scott, BTRL, Martlesham Heath, Ipswich, IP5 7RE, UK ++44 (473) $646863 / 646885$

$1-6$

Travemünde, Germany

8th Int. Conf, on Fourier Transfrom Spectroscopy

Ges. Deutscher Chemiker, Abteilung Tagung, Postfach 9004 40, D-6000 Frankfurt/Main 90 Fed. of European Chem. Socs.

$1-6$

Praha, Czechoslovakia

Mesons and Light Nuclei $V$

E. Truhlik, Inst. of Nuclear Physics,

CS-25068 Rez u Prahy

++42 (2) $844241 / 6857567$

A: 1 Jun 91 / PP / 100/SFR 230.-, incl. proc.

$2-4$

Nonlinear Guided Wave Phenomena '91

Meetings Dept., Optical Society of America,

2010 Massachusetts Ave.

Washington DC. 20036, USA

$++1(202) 2238130 / 2231096$

$2-4$

Cannes, France

Int. Symp. on Radars \& Lidars

P. Granes, Obs. de la Côte d'Azur, Dpt. CERGA av. Copernica, F-06130 Grasse

++33 () $93365849 / 93368963$

$2-64$

Edinburgh, UK

CM: Int. Conf. on Magnetism

C. Jones, The Institute of Physics,

47 Belgrave Sq., London, SW1X 80X, UK

++44 (71) $2356111 / 2596002$

A: 12 Jul 91 / Ab: 11 Mar 91 / PP: 31 May 91 /

1000 / f 245. - ; 134. - students (ex. proc.)

before 2 Sep 91 , incl. proc. / IUPAP
2 - 6 Sept.

Caen, France

Congrès de la Société Française de Physique

- Histoire des Sciences

Pour et par les Enseignants du Secondair

Phsique des Particules Elementaires

Confinement Inertie

Cances da Physique Atomique et

Les Plasmas Naturel

Physique du Micromètre au Nanomètre

Imperfections dans les Solides

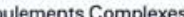

R. Bougault, LPC/ISMRa, bd du Maréchal-Juin, F-14032 Caen Cédex

++33 () $31452546 / 31473111$

Bitnet: sfpcaen @ caevax.decnet.cern.ch

A: 30 Apr 91 / Ab: 20 May 91 / NP / 700

French only / FF 900.-; 750.- IOM's

\section{2-6 Lake Balaton, Hungary}

European Research Conf. in Physics: Nuclear Shapes

J. Hendeković, ERC Programme, ESF 1, quai Lezay Marnésia,

F-67000 Srasbourg

++33 () $88255747 / 88366987$

2- 6

Int. Conf. ion Polymer-Solid Interfaces

Namur, Belgium

J.J. Pireaux, FUNDP, LISE, Faculté N.-

rue de Bruxelles, B-5000 Namu

++32 (81) $724595 / 230391$

- 6 b: 15 May 91

'91: 2nd European Cont, Nice, France Diamond-like and Related Coatings

COSMT, Conf. Organizers, 52, av, de la Gare, POB 415 $\mathrm{CH}-1001$ Lausanne 1

++41 (21) $234886 / 234972$

$3-6$ A: $19 \mathrm{Apr} 91 / \mathrm{PP}$

Adriatico Res. Conf.: Path Integration and

Trieste, Italy its Application

Adriatico Res. Conf., ICTP, POB 586, 1-34100 Trieste ++39 (40) $224241 / 22453$

3- 6 A: 15 Jun $91 / 70$ / inv. / ICTP, SISSA

Renormalisation Group 91

Dubna, USSR

D. Shirkov, Pres, Org. Comm., JINR Dubna, POB 79 Head Post Office, SU-101000 Moscow ++7 (095) $9262251 / 2002283$ tkatchev@ vxcern.cern.ch

A: 1 Feb 91 / Ab: 1 Apr 91 / PP: 1 Oct $91 / 100$ \$US 100.-

3 - 7

Frankfurt, Germany

ECAART 2: 2nd European Conf, on Accelerators in Applied Res. and Tech.

K. Bethge, ECAART 2, Nuclear Physics Inst., Univ. of Frankfurt, August-Euler-Str. 6 W-6000 Frankfurt/Main 90

++49 (69) $7984242 / 7984212$ Ab: 30 Apr 91 / PP: 2 Sep 91 / DM 430.-

$4-6$ 480. - after 1 Jul 91); 120. - students ex. proc

$4-6$ Lund, Sweden SES-II: Int. Worshop on Auger Spectroscopy and Electronic Structure (Satellite to ECOSS-12)

Malmö Convention Bureau, Mäster Johansgatan 6 , S-211 21 Mälmo

$++46(40) 232550 / 232560$

Ab: 15 May 91 / PP: 30 Aug 91 / SKR 1900.1900. - after 15 may 91 / IUPAP, SPS

Molecular Motion and Structure in Disordered Condensed Matter

J.H. Strange, Physics Lab., The University, Canterbury, Kent, CT2 7NR, UK

++44 (227) $764000 / 762616$

A: $15 \mathrm{Jul} 91$ / Ab: $15 \mathrm{Jul} 91 / \mathrm{NP}$ sUS $125,-: 85$ - students, incl, board, lodge British Radiofrequency Spectroscopy Group

4- 7 Krakow, Polan

ECIFUAS-6: 6th European Conf. on Internal Friction and Ultrasonic Attenuation in Solids

L.B. Magalas, Acad. of Mining and Metallurgy, Inst. of Metallurgy, Al. Mickiewicza 30 PL-30059 Krakow

$++48(12) 333823 / 331014$

Ab: 30 Mar 91/PP / SUS 250 - 150 - students

7 - 9 ex. proc. (280. - ; 180. - after 1 Jun 91), incl. proc.

2nd Symp. on High Performance Computing USC, SHPC Secrétariat, rue de St. Priest, 950 , BP 7229, F-34184 Montpellier Cédex ++33 () $67141414 / 67523763$

8 - $14 \quad$ Jaca, Huesca, Spain

NATO Adv. Res. Workshop: New Trends in Nonlinear Dynamics - Nonvariational Aspects

C. Perez-Garcia, Univ. de Navarra, Dept. of Physics, E-31080 Pamplom

9 -11 Sept.

München, Germany

(th Symp on Turbulent Shear Flows

FW. Schmidt, Dept. of Mech Engng, Penn. State Univ. University Park, PA 16802, USA

$++1(814) 8652072 / 8634848$

- 11

Ab: 15 Nov 90 / PP: 31 May 91

- 11 European Conf on Electron \& Optical Beam of Integrated Circuits

9 - 12

- 12 Montpellier, France Their Effects on Devices \& Systems

J.P. Charles, RADECS, CEM, Case 083, Univ. de Montpellier, F-34095 Montpellier Cédex 05 ++33 () $67525633 / 67143810$

Stockholm, Sweden

ECOSS-12: 12th Europ. Conf. on Surface Science

ECOSS-12, c/o CONGREX, Box 5619 ,

S-114 86 Stockholm

++46 (8) $6126900 / 6126292$

Ab: 30 Apr 91 / PP: 26 Aug 91 / SKR 2000.-

1800. - IOM's; 1400 . - students

(2200. - ; 2000. -; 1500. - after 31 May 91)

9 - 12

Villigen, Switzerland

4th Int. Workshop on Targetry \& Target Chemistry

R. Weinreich, c/o PET Progr., PSI, CH-5232 Villigen ++41 (56) $993686 / 993294$

$9-12$ Kleinheubach, Germany

WE Heraeus Seminar and

EPM-3: 3rd Int. Workshop on Electronic Properties of Metal/Non-Metal Microsystems

H. Lehmberg, Inst. f. Angewandte Physik,

TH Darmstadt, Schlossgartenstr. 7,

W-6100 Darmstadt

A: 1 Aug $90 / \mathrm{Ab}: 5 \mathrm{Apr} 91 / \mathrm{PP}$ /

- 12

DM 250.- incl. proc

Symp. on Titan

JP. Lebreton, ESA Space Sci. Dept. ESTEC POB 299 NL-2200 AG Noordwijk

$++31(1719) 83600 / 84697$

Span: estcs1 @ jlebreto

- 13

Ab: 15 Jun 91 / A: 9 Sep 91

Glasgow, UK Deposition

M.L. Hitchman, Univ. of Strathclyde,

Glasgow, Scotland, UK

$9-13$ ++44 (41) $5524400 / 5520775$

Int. Conf. on Synchrotron Radiation Grenoble, France Phenomena

C. Troyanowsky, Soc. de Chimie Physique

10, rue Vauquelin, F-75231 Paris Cédex

++33 (1) $47075448 /$

SFC, AICF, DBGPC, RSC

$9-13$

11th Int. Scientific Instrument Symp.

G. Dragoni, Museo di Fisica, via Imerio, 46

1-40126 Bologna

++39 (51) $351099 / 247244$

A: 1 Apr 91 / Ab: 1 Jun 91 / LIT 120000.-

CNR, Int. Union of Hist. \& Phil. Sci.

$9-13$

J. Moret-Bailly, Lab. de Spectronomie Mol. et Inst. Laser (S.M.I.L.), 6, bd. Gabriel, F-21100 Dijon

++33 () $80396000 / 80395066$ smil @ frccub11 French

9- 13

Int. Conf, on Gravitationat Lenses

Hamburg, Germany

R. Kayser, Hamburger Sternwarte

$-13$ ojenbergsweg 112, W-2050 Hamburg 80

Toledo, Spain 
9- 17 Sept.

Varenna, Italy

High Power Microwave Generation and Applications

E. Sindoni, ISPP, Dip. Physica, 16 via Celoria, 1-20133 Milano

++39 (2) $2392267 / 2392205$

A: 15 Jun 91 / Ab: 15 Jun 91/PP: 17 Sep $91 / 100 /$

LIT 500000. - ; 200000. - students, incl. proc. /

0 - 12 PPP, ENEA, CEC, CNR

Nuclear Structure at Storage Rings: D Nuclei and Subthreshold Meson Essembly

P. Arve, Dept. of Mathematical Physics, Lund Inst. of Technology, POB 118, S-22 100 Lund ++46 (46) $109090 / 104416$ - 13

Bristol, UK

EMAG '91: Electron Microscopy and Analysis Conf.

S. Lippmann, The Institute of Physics, 47 Belgrave Sq., London, SW1X 80X, UK $++44(71) 2356111 / 2596002$ A: 9 Aug 91 / Ab: 28 Mar 91 / PP: 10 Sep 91 f 200.-, incl. proc. / IOP

$10-15$

Beijing, PRC

IMEKO-XII: 12th IMEKO World Congress

IMEKO XII Secretariat, Chinese Soc. for Measurement, POB 1413, Beijing 100013, PRC ++86 (1) $444304 / 898116$ $\mathrm{Ab}: 30$ Oct $90 / \mathrm{PP}$

$11-13$

SMM 10: Soft Magnetic Materials 10

M. Müller, SMM 10, Zentralinstitut f.

Feskörperphysik und Werkstofforschung,

Helmholtzstr. 20, 0-8027 Dresden

++37 (51) $4659322 / 4659600$

A: 1 Jul 91 / Ab: 31 Jan 91 / PP /

DM 650.-; 500.- IOM's; 100.- students

11 - 13

Milano, Italy

CSCIRC' 91: 17th European Solid State Circuits Co

++39 (2) $744149 / 7385567$

Ab: 10 Apr 91 / PP / LIT 450000 .

(525000.- after 15 Jul 91); 150000 . - students

15 - 19 nr. Praha, Czechoslovakia

European Research Conf.: Physical Metallurgy Grain Boundary, Interface \& Mechanical Properties

J. Hendeković, ERC Programme, ESF,

1, quai Lezay Marnésia, F-67000 Srasbourg

++33 () $88255747 / 88366987$

A: 22 Apr 91 / NP / $80 / C E C$, ESF

$15-20$

Amsterdam, The Netherlands

8 th Int. Conf. on Secondary lon Mass Spectrometry

RAI Organisatie Bureau Amsterdam, Europaplein 12, NL-1078 GZ Amsterdam

16 - 18
3rd Int Conf. Holographic Systems, Components and Applications

IEE Conf. Services, Savoy Place, Victoria Embankment, London, WC 2R OBL, UK

++44 (71) $2401871 / 2407735$

Ab: 22 Feb 91 / PP: 31 May 91

16 - 19 Heidelberg, Germany

INCMRM-1: 1st. Int. Conf. on NMR Microscopy

W. Kuhn, MPI Polymerforschung, Postfach 3148

W-6500 Mainz

Ab: 30 Jun 91/DM 600.-, 700.- after 16 Sep 91

$16-19$ Montreux, Switzerland

ESSDERC: European Solid State Device Res. Conf.

$\mathrm{H}$. llegems, Inst. de Micro-optoélectronique, Dépt. de Physique, EPFL, CH-1015 Lausanne ++41 (21) $6933441 / 6934444$ Ab: 15 Mar 91

$16-20$

Mandelieu-Cannes, France

Coll.: Matériaux dans l'Environnement Spatial

Dépt. Coll., CNES, 18, av Edouard-Belin, F-31055 Toulouse Cédex

++33 () $61273131 / 61281327$ French / CNES, CERT, ESA

16 - 20

Wien, Austria

8th Int. Conf. on Modern Trends in Activation Analysis

F. Grass, Atominstitut, Schüttelstr. 115, A-1020 Wien Ab: 31 May 91 / PP: 16 Sep 91 /

SCH 3500.-, 4000.- after $15 \mathrm{Jul} 91$, incl. proc.

16 - 20

Hadron Structure ' 91

R. Lietava, Physical Inst. of SAV, Dúbravská cesta 9, CS-84228 Bratislava - 19

Krakow, Poland

Physics for Industry - Industry for Physics

A. Oles, Inst. of Physics and Nuclear Techniques, Acad. of Mining and Metallurgy,

Al. Mickiewicza 30, PL-30 059 Krakow

++48 (12) $344482 /-$

Ab: 1 Apr 91 / $300 /$ \$US 300. - ; 100. - students (350.- ; 125. - after 1 Jun 91)

18 - 20

Kiel, Germany

Workshop on Atmospheres of Early Type Stars

S. Jeffery, Dept. od Physics \& Astronomy, St. Andrew's Univ., North Haugh, St. Andrews, Fife, KY16 9SS, UK $++44(334) 76161 / 74487$
18 - 20 Sept.

London, UK

Faraday Disc. 92: The Chemistry and Physics of Smal Metallic Particles

A. Fish, Royal Society of Chemistry,

Burlington House, London, W1V OBN, UK

++44 (71) $4378656 / 7341227$

A: 31 Aug 91 / Ab: 30 Aug 90 / PP: 31 May $91 /$ 200 / f 80. - ; 10. - students

$22-25$

5th Conf. on Sensors and Their Applications

J. Butler, The Institute of Physics,

47 Belgrave Sq., London, SW1X 80X, UK ++ (71) $2356111 / 2596002$

A: 1 Aug 91 / Ab: 8 Apr 91 / PP: 8 Apr 91 f 180.-, incl. proc.

22 - 27

Cambridge, UK

NATO Adv, Res. Workshop: The Theory of Sunspots

J.H. Thomas, Dept. of Physics \& Astronomy, Rochester, NY 14627, USA

$22-27$

Salzburg, Austria

NRE-V: 5 th int. Symp, on the Natural Radiation Environment

M. Olase, CEC, DG XII/D/3, 200, rue de la Loi, B-1049 Bruxelles

23 - 25

Int. Workshop on Fast Wave Current Drive in Reactor-Scale Tokamaks

D. Cribier, CEN, F-91191 Gif-sur-Yvette Cédex A: 15 Apr 91 / Ab: 15 Jun 91 / PP: 23 Sep $91 / 50$

$23-25$

8. Tagung Mikrosonde

A. Röder, Sektion Physik der Univ.

Friedmann-Bach-Platz 6, 0-40

$23-25$

Leipzig, Germany

Coll.: Horizons de l'Optique - Optique Spatiale

CNES - Délégation à la Communication,

18, av. Edouard-Belin, F-31055 Toulouse Cédex

++33 () $61273131 / 61281327$

French / CNES, SFO

23- 25

Saclay, France of the Nucleon

H.P. Morsch, Lab. National Saturne, CEN Saclay,

F-91191 Gif-sur-Yvette Cédex

++33 (1) $69086196 / 69089011$

amorsch@frcpnaa

$23-26$

Dubna, USSR

5 th EBIS Symp

E.D. Donets, JINR, Headpostoffice Box 79 SU-101000 Moscow

ISE 7: 7th Int. Electret Symposium

D. Geiss, Academy of Science, Inst. of Polyme Chemistry, "Erich Correns", Kantstr. 55, O-1530 Teltow

$23-27$ 27

Spanish Physical Soc. 23rd Biennial Meeting
J. Casanova, Fac. de Ciencias, E-47071 Vall

Valladolid, Spain ++34 (83) 250458 st. $2215 / 257293$

$23-27$ Vall

J. Casanova, Fac. de Ciencias, E-47071 Valladolid

++34 (83) 250458 ext. $2215 / 257293$

$23-27$

Graz, Austria

Austrian Phys. Soc. 41st Ann. Meeting

H. Latal, Inst. f. Theo. Physik, Univ. Graz, Universitätsplatz 5, A-8010 Graz ++43 (316) $3805230 / 384091$

utp @ dvz.uni-graz.ada.at

A: 1 Sep 91 / Ab: 15 May 9 / NP / 300 / German / AS 400. - students free,

23- 27

Workshop on Polized Heidelberg, Germany

Polarized Gas Targets for Storage Ring W-6900 Heidelberg 1

++49 (621) $516408 / 516540$

Bitnet: est @ cruxnhd3

23- 27 Heraklion, Crete, Greece

27th Europhys. Conf. on Macromolecular Physics: Applns of Scattering Methods to the Dynamics of Polymer Systems

G. Fytas, FORTH - IESL, POB 1527

GR-711 10 Heraklion

++30 (81) $239757 / 239735$

fytas@grcrvax

A: 31 Jan 91 / PP / 150 / \$US 150.-

100. - students, incl. proc.

$23-28$

7 th Int. Workshop on Room Temperature

Semiconductor X-ray \& Gamma-ray Detectors \& Associated Electronics

E. Perillo, Ravello Workshop, c/o Dip. di Sci. Fisiche, pad. 20 Mostra d'Oltremare, 1-80125 Napoli

++39 (81) $7253315 / 614508$

Ab: 15 May 91 / PP / LIT 400000.-

CE, E-MRS, CNR

24 - 27 Sept.

Giardini Naxos, Sicily, Italy

Int. Meeting on Opcitons in Confined Systems

A. Quattropani, Inst. de Physique Théorique,

PHB-Ecublens, CH-1015 Lausanne

++41 (21) $6933421 / 6934444$

Bitnet: quattro @ eldp.epfl.ch

PP

$24-27$

Leningrad, USSR

14th Int. Conf. on Coherent \& Nonlinear Optics

S.A. Akhmanov, Physics Dept., Moscow State Univ., Leninske Gory, SU-119899 Moscow ++7 (095) $9390125 / 9390126$

$25-27$

Toulouse, France

ICSO 91: Int. Conf. on Space Optics

CNES - Délégation à la Communication,

18, av. Edouard-Belin, F-31055 Toulouse Cédex ++33 () $61273131 / 61281327$ French

26 - 28

Thessaloniki, Greece

Balkan Physical Union 1st Gen. Conf.

E.K. Polychroniadis, Dept. of Physics 313-1, Aristotle Univ. of Thessaloniki, GR-54006 Thessaloniki ++30 (31) $992715 /$ -

412181

A: 30 Apr 91 / Ab: 30 Apr 91 / PP: 30 Apr 91 / 1000 / \$US 150.-; 20.- IOM's; 10.- students

27 - 30

Physical Soc. of Japan 46th Ann. Meeting

Y. Iwaya Kikai-Shinko Building, 3-5-8 Shiba-Koen, Minato-ku, Tokyo 105, Japan

++81 (3) $34342671 / 34320997$ A: 20 Jun 91 / Ab: 6 Aug 91 / NP / 3300

Japanese / Y 2060. - ; 1030. - students

28 Sept. - Oct.

Int. Conf. on Education in Optics

$\mathrm{H}$. Wilrich, SPIE, Lennestr. 55, W-5300 Bonn 1

++49 (228) $219062 / 219066$

Ab: 1 Feb 91 / PP / SPIE, OSA

29 Sept. - 3 Oct.

European Research Conf, in Physics: Quantum Optics

J. Hendeković, ERC Programme, ESF 1, quai Lezay Marnésia.

F-67000 Srasbourg

++33() $88255747 / 88366987$

30 Sept. -3 Oct.

Autrans, France

European Research Conf, in Physics: Dynamic Properties of Solids

J. Hendekovic, ERC Programme, ESF, 1, quai Lezay Marnésia,

F-67000 Srasbourg

++33 () $88255747 / 88366987$

30 Sept. - 4 Oct.

Bensheim, Germany

ICIS 91: 4th Int. Conf. on Ion Sources

B. Wolf, GSI Darmstadt, Postfach 110552

W-6100 Darmstadt

++49 (6151) $359320 / 359785$

Bitnet: ul31@ddagsi3

Ab: 30 Mar 91 / PP: 30 Aug 91 / DM 440.

(540. - after 30 May 91); 125. - students

(ex. proc.), incl. proc. / AVS, IUPAP

30 Sept. - 4 Oct. Particle Physics

Int. Centre for Theo. Physics, POB 586, I-34100 Trieste ++39 (40) $22401 / 224163$

30 Sept. -4 Oct.

Darmstadt, Germany

3rd. Int. Symp.: Spacecraft Flight Dynamics

R.E. Munch, ESO Centre, Orbit Altitude Div.

Robert-Bosch-Strasse 5, W-6100 Darmstadt 
7- 11 Oct.

Bayreuth, Germany

UPS 1991: Int. Symp. on Ultrafast Phenomena in Spectroscopy

A. Laubereau, Univ. of Bayreuth, POB 101251

W-8580 Bayreuth

$-1++49$ (921) 552999

A: 7 Oct $91 /$ PP: 1 Jun $91 / 250$ /

DM 250. -; 150. - students, incl. proc

7- 11

Trieste, Italy

Workshop on Stochastic and Deterministic Models

Int. Centre for Theo. Physics, POB 586, 1-34100 Trieste $++39(40) 22401 / 224163$

A: 31 Mar 91

$8-10$

Nice, France

Int. Conf. on Microwave and High Frequency

Comité Français de l'Electricité, Tour Atlantique, F-92080 Paris-La Défense Cédex 06

8- 12 + 33 (1) $47736615 / 47739553$

Richmond, VA, USA

int. Symp. on the Physics and Chemistry of Finite Systems - From Clusters to Crystals

P. Jena, Physics Dept., Virginia Commwealth Univ.,

Richmond, VA 23284-2000, USA

$++1(804) 3671313 / 3678599$

Bitnet: khanna @ vcuax

Ab: 15 Jun 91 / \$US 250. - before 15 May 91; 295. - after

9 - 12

Zürich, Switzerland

SUPERLEAR Workshop

C. Amsler, Physik-Inst., Univ. Zürich,

Schönberggasse 9, $\mathrm{CH}-8001$ Zürich

$++41(22) 7672914 /$

Bitnet: amsler@ atcernvm.cern.ch

10 - 14 Davos, Switzerland

European Research Conf. in Physics:

Fundamental Aspects of Surface Science

J. Hendeković, ERC Programme, ESF

1. quai Lezay Marnésia,

F-67000 Srasbourg

++33 () $88255747 / 88366987$

14-18 Amsterdam, The Netherlands

EWEC '91: European Wind Energy Conference

Van Namen \& Westerlaken, Cong. Org. Services,

POB 1558, NL-6501 BN Nijmegen

$4-18$

Budapest, Hungary
ECASIA 91: 4th Eur. Conf. on Applications of Surface and Interface Analysis

L. Köver, Inst. of Nuclear Res. of the Hungarian Acad. of Sci., MTA ATOMKI, Pf. 51, H-4001 Debrecen $++36(52) 16181 \%$ 72210 atom $\mathrm{h}$

PP / MTA ATOMIKI, Aust. Soc. for Microchemistry

$14-18$

Microchemistr

7th Symp. on Neutron Dosimetry

R. Jahr, 7th Symp. on Neutron Dosimetry, Abt. 7. PTB, Bundesallee 100, W-3300 Braunschweig ++49 (531) $5927010 / 5924006$ Ab: 15 Feb $91 /$ PP: 14 Oct $91 /$

DM 380.-, incl. proc. / CEC, PTB, DoE

14 - 18

Capri, Italy

2nd Int. Conf. on Calorimetry in High Energy Physics

A. Ereditato, INFN Napoli, Mostra d'Oltremare, PAD. 20, 1-80125 Napoli

++39 (81) $7253240 / 614508$

ereditat@ cernvn

A: 15 Jul 91 / Ab: 15 Aug 91 / PP / $180 /$ LIT 200000.-, incl. proc.

$14-18$

Strasbourg, France

DYMAT-3: 3rd Int. Conf. on Mechanical \& Physical Behaviour of Materials Under Dynamic Loading

Est. Tech. Central de l'Armement, 16 bis, av. Prieur de la Côte d'Or, F-94114 Arceuil Cédex Ab: 1 Oct 91 / A: 15 Apr 91 / French

14 - 19 Château de Blois, France

Frontiers of Life: 3 e Rencontre de Blois

J. Tran Thanh Van, Bât. 211, Univ. de Paris-Sud, F-91405 Orsay Cédex

++33 (1) $69285135 / 69288659$ trantv@ frcpn11

$21-25$

Thessaloniki, Greece

Hellenic Phys. Soc. 29th Ann. Scientific Meeting

G.A. Kourouklis, Physics Div., School of Technology, Univ. of Thessaloniki, GR-54006 Thessaloniki ++30 (31) $992612 / 992613$

28-31 Kreuth, Germany

European Research Conf, in Physics: Very High Resolution Spectroscopy with Photoelectrons - ZEKE Spectroscopy

J. Hendekovic, ERC Programme, ESF,

1, quai Lezay Marnésia,

F-67000 Srasbourg

++33 () $88255747 / 88366987$
3 - 8 Nov

OPTCON '91: OSA Annual Meeting

Washington 20036 , USA

$$
++1(202) 2238130 / 2231096
$$

3-8 San Jose, CA, USA

Symp. on Optical Sci. and Engineering

SPIE, POB 10, Bellingham, WA 98227-0010, USA $++(206) 6763290 / 6761445$
Ab: 8 Apr $91 / \mathrm{A}: 70 \mathrm{Ot} 91 / \mathrm{SPIE}$

4-8

Strasbourg, France

5 th Int. Symp. on the Scientific Basis for Nuclear Waste Management

A. Saas, Dept. of Waste Management \& Disposal, CEN Caradache, F-13108 St. Paul Lez Duranz ++33 () $42252720 / 42254646$ Ab: 30 May 91 / PP / FF 2000,-, incl. proc. MRS, E-MRS, CEA

4-8

Strasbourg, France

European Materials Res. Soc. Fall Meeting

P. Siffert, E-MRS, C.T.T., BP 20,

F-67037 Strasbourg Cédex

++33 () $88286543 / 88280990$

Ab: 1 Jul 91 / PP / FF 2000. - ; 900

(2200.-; 1100.- after 20 Sep 91), incl. proc.

$10-15$

San Francisco, CA, USA

American Nuclear Soc. Winter Meeting

American Nuclear Society, 555N. Kensington Ave.,

La Grange Park, IL 60525, USA

$++(708) 3526611 / 3526464$

$11-15$

Tsukuba, Japan

ICALEPCS '91: Int. Conf. on Accelerator and Large Experimental Physics Control Systems

S. Kurokawa, KEK, 1-1 Oho, Tsukuba-shi,

Ibaraki-ken, 305 Japan

++81 (298) $641171 / 643182$

kurokawa @ jpnkekvm

$\mathrm{Ab}: 31$ Mar 91 / PP: 11 Nov 91 /

Y 25000.-, incl. proc.

$11-15$

QM '91: Quark Matter Conf.

Gatlinburg, TN, USA

F. Plasil, Bldg 6003, POB 2008, MS-6327, Oak Ridge TN 37831-6372, USA

$++1(615) 5744711 / 5762822$ Bitnet: qm91 @ orph01

13-15 Amsterdam, The Netherlands

Workshop on Molecule - Surface Interactions

C.M. van den Heuvel, Royal Acad. of Arts and Sciences, Postbus 19121, NL-1000 GC Amsterdam ++31 (20) $6220229 / 6204941$

$13-16$

London, UK

QSA-6: Int. Conf. on Quantitative Surface Analysis

G.C. Smith, NPL, Div. of Materials Tech. Teddington, Middlesex, TW11 OLW

Ab: 25 Aug $90 / P P / f 241.50$

Int. Conf. on Molcule - Surface Interactions

Conference Service, KFA Jülich, Postfach 1913, W-5170 Jülich

$-1++49$ (2461) 614666

A: 31 Aug $91 /$ Ab: 30 Jun 91/PP: 14 Oct 91 DM 100. - ; 50. - students, incl. proc.

(1) Garching, Germany

High Resolution Imaging by Interferometry II

Interferometry '91, c/o C. Stoffer, ESO, Karl-Schwarzschild-Str. 2, W-8046 Garching bei München

18- $21 \quad$ Bournemouth, UK

ANN 91: 2nd Int. Conf. on Artificial Neural Networks

ANN 91, IEE Conf. Services, Savoy Place,

Victoria Embankment, London, WC2R OBL, UK

++44 (71) $2401871 / 2407735$

Ab: 28 Mar $91 /$ PP. 31 Jul 91

$18-22$

Villepinte, Paris, France

Exposition de Physique

M. Veyssié, Sec. Gén., Soc. Française de Physique, 33 , rue Croulebarbe, F-75013 Paris ++33 (1) $47073298 / 43317426$ French

$19-22$

Ajaccio, France

Coll.: Le Comportement des Fluides dans I'Espace

CNES - Délégation à la Communication, 18, av. Edouard-Belin, F-31055 Toulouse Cédex ++33 () $61273131 / 61281327$ French

20-22

Toulouse, France

Int. Symp. on Correlation

Scientific Secretariat, Acad. Nat. de l'Air et de I'Espace, 1 , av. Camille Flammarion, F-31500 Toulouse ++33 () $61581526 / 61263756$ French

24- 27

Kyoto, Japan

ICNF 91: 11th Int. Conf. on Noise in Physical Systems and $1 /$ f Fluctuation

T. Musha, Tokyo Inst. of Tech., Nagatsuta, Midori-ku, Yokohama 227, Japan

++81 (45) 9221111 ext. 2546/921 1204

Ab: 28 Feb 91 / PP: 31 Aug 91
25 - 29 Nov.

Strasbourg, France

3rd Int. Symp. on Trends \& New Applns in Thin Films

J. Fauvet, SfV, 19, rue du Renard, F-75004 Paris ++33 (1) $42781582 / 42786320$ Ab: 15 Apr 91 / PP: 5 Sep 91 / 200 /

FF 2500. - ; 1500. - student (3000. - ;

1800 - after 20 May 91), incl, proc. / SfV, DAGV

2-6 Dec.

Materials Res. Soc. Fall Meeting

M. Geil, Materials Research Soc., Meetings Dept. 98000 McKnight Rd., Pittsburg, PA 15237, USA ++1 (412) $3673003 / 3674373$ Ab: 1 Jul 91

$3-5$

Berlin, Germany

11. Tagung "Physik und Elektronik"

R. Keiper, Sektion Physik der Humboldt-Univ., Invalidenstr. 110, 0-1040 Berlin

9- 12

Shimoda, Japan

Symp.on Hypernuclear and Strange Particle Physics

3-2-1, Midori-cho, Tanashi, Tokyo 188

++81 (424) 614131 ext. $222 / 620763$

Bitnet: hyper91 @ jpnutins

A: 31 Oct $90 / 130$

$10-13$

Sydney, Australia

Fibres in Astronomy II

$16-18$

y, Australia

16- 18 Southampton, UK

The Conformations of Flexible Molecules in Fluid Phase

G.R. Luckhurst, Dept. of Chemistry, The Univ.,

Southampton, SO9 $5 \mathrm{NH}$, UK

++44 (703) $593795 / 59378$

Ab: 15 Nov 91 / PP: 15 Aug $91 / 120 /$

f 80. - ; 20.-

17 - 18

oll. COMET 6: Sciences des Matériaux en Microgravité

CNES - Délégation à la Communication,

18, av. Edouard-Belin, F-31055 Toulouse Cédex

++33 () $61273131 / 61281327$

French

Birmingham, UK

Ann. Condensed Matter and Materials Physics Conf.

C. Gough, School of Physics and Space Research,

Univ. of Birmingham, Edgbaston,

Birmingham, BI5 2TT, UK

++44 (21) $4144669 / 4146709$

$\mathrm{PP} / 800$

Conferences 1992

\section{2 \\ European Macromolecular Physics \\ A. Mjasnikova, A.F. loffe Physico-Technical Inst., USSR Acad. of Sciences, K-21, Politechnicheskaya 26 SU-194021 Leningrad}

1992

UK

X-Ray Diffraction: Applications and Materials Science M. Gee, Div, of Materials Applications, Nat. Physical Lab., Teddington, Middlesex, TW11 OLW, UK 1992

Ann. Gen. Conf. of the Int. Optical League Int. Optometric and Optical League,

10 Knaresborough PI., London, SW5 OTG, UK

Jan.

Coll.: Astronomie des Hautes Energies

CNES - Délégation à la Communication,

18, av. Edouard-Belin, F-31055 Toulouse Cédex

$19-24$

OE/LASE '92: Optics, Electro-Optics and

Laser Applications in Science \& Technology

SPIE, POB 10, Bellingham, WA 98227-0010, USA

3-6 Feb. 


\section{9- 13 March}

Karisruhe, Germany

Int. Symp. on Applications of Isotopes \& Radiation in Protection of the Environment

IAEA, Conf. Service Sect., POB 100, A-1400 Wien

12 - 16 Aussois, France

Int. Workshop on Dynamical Fluctuations \& Correlations

E. Suraud, GANIL, BP 5027, F-14021 Caen Cédex

$16-20$ Indianapolis, IN, USA

APS March Meeting

American Physical Soc., 335 E 45th St. New York, NY 10017

$16-20$

Regensberg, Germany

Frühjahrstagung des Arbeitskreises Festkörperphysik

- Dünne Schichten

- Dynamik und Statistische Physik

- Halbleiterphysik

- Magnetismus

Tiefe Temperaturen

- Vieke Temperaturen

DPG-Ges., Haupstr. 5, W-5340 Bad Honnef

$20-27$

Somerset, NJ, USA

Adv. in Semiconductors \& Superconductors

Physics Toward Device Applications

SPIE, POB 10, Bellingham, WA 98227-0010, USA

$23-27$

Antibes, France

Interdisciplinary Conf. on Dielectric Surfaces

$\mathrm{SfV}, 19$, rue du Renard, F-75004 Paris

23-27 Hannover, Germany

Frühjahrstagung der Fachausschüsse

- Atomphysik

- Kurzzeitphysik

Molekülphysik

Oberflächenphysik

Plasmaphysik

- Quantenoptik

DPG-Ges., Haupstr. 5, W-5340 Bad Honnef 1

\section{3- 28 \\ Berlin, Germany \\ 3rd EPAC: European Particle Accelerator} Conference

Ch. Petit-Jean-Genaz, CERN, LEP Division, $\mathrm{CH}-1211$ Geneva 23

47th Ann. Meeting of the Physical Soc. of Japan

Y. Iwayanagi, The Physical Society of Japan, Room 211, Kikai-Shinko Building, 3-5-8 Shiba-Koen,

Minato-ku, Tokyo 105, Japan

\section{Schools 1991}

2- $12 \mathrm{May}$

Erice, Italy

Int. School on Quantum Electronics: 16th Course - Phase Transitions in Liquid Crystals

A. M. Scheggi, IROE-CNR,

via Pianciatichi, $64,1-50127$ Firenze

$++39(55) 4378512 / 4379569$

A: 2 Mar 91 / PP / 80 / \$US 800. - , incl. board,

$$
2 \text { - } 4
$$$$
\text { lodge, incl. proc. / NATO, CNR }
$$

Cargese, Corsica, France

Journées Relativistes

D.A. Folacci, Fac. des Sciences, Univ. de Corse, BP 52 , F-20250 Corte

++33 () $95450034 /-$

jensen @ frmop22

A: 15 Mar 91 / Ab: 1 Feb 91 / PP / 70 / French / CNRS

$5-18$

JINR-CERN School of Physics

Alushta, Crimea, USSR

S.M. Tracy, CERN School of Physics, CERN-DG,

$\mathrm{CH}-1211$ Geneva 23

++41 (22) $7672724 / 7823011$

tracy @ cernvm.cern.ch

A: 30 Jan 91 / limited to 100 / inv. /

SFR 1300.-, incl. board, lodge

6 May - 7 June

Trieste, Italy

Spring College in Materials Sci. on Nucleation, Growth and Segregation in Materials Science and Engng.

Int. Centre for Theo. Physics, POB 586, I-34100 Trieste ++39 (40) $22401 / 224163$

A: 30 Nov 90

$12-22$

$22 \quad$ Erice, Italy 12th Course: Black Hole Physics

V. de Sabbata, Inst. di Fisica dell'Univ., via Imnerio, 46, I-40126 Bologna

A: 15 Feb 91 /PP / SFR 1500.--, incl. board, lodge, incl. proc. / NATO

20- 31

3rd ICFA School on Instrumentation in Elementary Particle Physics

Int. Centre for Theo. Physics, POB 586, 1-34100 Trieste ++39 (40) $22401 / 224163$

A: 28 Feb 91 / limited / inv. / ICFA, INFN
21 May

Brussels, Belgium

An Introduction to lon Assisted Processes and

Their Applications (Satellite to IPAT 91)

IPAT Secretariat, 26-28 Albany St.

Edinburgh, $\mathrm{EH} 13 \mathrm{OH}$, UK

++44 (31) $5572478 / 5575749$

Ab: 16 Nov 90 / PP: 15 Feb 91

BF 16800.-, incl. proc.

P. Siffert, E-MRS, C.T.T., BP 20

F-67037 Strasbourg Cédex

++33() $88286543 / 88280990$

A: 15 Ap 91 / limited to 25 / FF 3000 . 3300. - after 15 Apr 91

27 May - 21 June

Spring School on Plasma Physics

Trieste, Italy

Int. Centre for Theo. Physics, POB 586, I-34100 Trieste ++39 (40) $22401 / 224163$ A: 31 Mar 90 / limited

30 May - 9 June

Erice, Italy

Int. School of Crystallography - 18th Course: Static, Kinematic and Dynamic Aspects of Crystal and Molecular Structure

L. Riva di Sanseverino, Executive Secretary, Int. School of Crystallography,

Piazza Porta San Donato, 1, 1-40126 Bologna ++39 (51) $243556 / 243336$

t54bom12@icineca

A: 30 Nov 90 / SFR 1500.-, incl. proc. / luCr

1 - 10 June II Ciocco, Italy Supramolecular Aggregates and Strongly Interacting Colloids

S.H. Chen, Dept. of Nuclear Engineering, Massachusetts Inst. of Tech. Cambridge, MA 02139, USA

3- 14

Trieste, Italy

2nd School on Non-Accelerator Particle Astrophysics Int. Centre for Theo. Physics, POB 586, 1-34100 Trieste ++39 (40) $22401 / 224163$

3- $14 \quad$ Trieste, Italy

Working Party on Initiation and Growth of Cracks in Materials

Int. Centre for Theo. Physics, POB 586, I-34100 Trieste $++(40) 22401 / 224163$

3-7 Cargèse, Corsica, France NATO Adv. Res. Workshop: Nuclear Shapes \& Nuclear Structure at Low Excitation Energies

F. Dykstra, Inst. de Physique Nucléaire, F-91406 Orsay ++33 (1) $69417318 / 69416470$ dykstra@ fripn51

A: 28 Feb 91 / Ab: 21 Jan 91 / PP / 80 / inv. / French / CNRS - CEA

$4-14$

Zakopane, Poland

31st Krakow School of Theoretical Physics: Particle Physics at High Energy

W. Slominski, Inst. of Physics, Jagellonian Univ., ul. Reymonta 4, PL-30-059 Krakow A: 15 Apr 91 / \$US 250.-, incl. board, lodge

$9-15$ Cargèse, Corsica, France

Collective Acceleration in Collisionless Plasmas

A. Roux, CNET/CRPE,

F-92131 Issy-les-Moulineaux Cédex ++33 (1) $48863500 /-$

A: 1 Feb $91 / \mathrm{PP} / 60 /$ inv. / French / NATO, CNRS 10 June-16 Aug New Hampshire, USA

Gordon Research Conferences - Frontiers of Science

10- 14 June Condensed Matter Physics
$17-21$ June Liquid Crystals

Holography
Gravitational Effects

24-28 June Polymers

Thermosetting High Perf. Materials

Polymer Colloids

8-12 July Elastomers

Neural Plasticity

norganic Thin Films

Matrix Isolated Species

Origins of Solar Syster

15-19 July Magnetic Resonance

Laser Diagnostics in Combustion

Crystal Growth

22- 26 July Nuclear Physics

Nonlinear Optics \& Laser

29 July - 2 Aug. Coatings and Films

Solid State Sudies in Ceramics

Physical Metallurgy

Molten Salts \& Liquid Metals

Dynamics of Gas-Surface Interaction

Metal and Semiconductor Clusters

Solar Plasma \& MHD Processe

$\begin{array}{ll}12-16 \text { Aug. } & \text { X-Ray Physics } \\ \text { lon Containing Polymers } \\ \text { Science of Adhesion } \\ \text { Chemistry \& Physics of Liquids }\end{array}$

$\begin{array}{ll}12-16 \text { Aug. } & \text { X-Ray Physics } \\ \text { lon Containing Polymers } \\ \text { Science of Adhesion } \\ \text { Chemistry \& Physics of Liquids }\end{array}$

$\begin{array}{ll}12-16 \text { Aug. } & \text { X-Ray Physics } \\ \text { lon Containing Polymers } \\ \text { Science of Adhesion } \\ \text { Chemistry \& Physics of Liquids }\end{array}$

$\begin{array}{ll}12-16 \text { Aug. } & \begin{array}{l}\text { X-Ray Physics } \\ \text { lon Containing Polymers } \\ \text { Science of Adhesion } \\ \text { Chemistry \& Physics of Liquids }\end{array}\end{array}$

M. Cruickshank, Gordon Research Center, . Cruickshank, Gordon Research Center, Univ. of Rhode Island, Kingston, RI 02881-0801, USA ++1 (401) $7834011 / 7837644$

Bitnet: bcp101@uriacc

\$US 400.-, incl. board, lodge

10 - 15 June

Espoo, Finland

Supercomputing in Science \& Engineering: Simulation of Matter

K. Kankaala, CSC, POB 40, SF-02101 Espoo ++356 (0) $4572239 / 4572302$ kankaala@ csc.fi

11 - 17

INFN Eloisatron Project: 15thWorkshop -

Erice, Italy QCD at $200 \mathrm{TeV}$

G. Coletti, Dipt. di Mathematica, Univ., via Vanvitelli, 1, I-06100 Perugia ++39 (75) $5855001 / 5852067$ giulicol@ipguniv

A: 31 Dec 90 / \$US 700.-, incl. board, lodge Trieste, Italy

Miniworkshop on Nonlinearity, Fractals and Pattern Formation

Adriatico Summer Prog. on Complex Systems, ICTP, POB 586, I-34100 Trieste ++39 (40) $224241 / 224531$ A: 30 Jan 91

$13-27$

Les Houches, France

NATO Adv. Study Inst.: Infrared Astronomy with ISO T. Encrenaz, Dépt. des Recherches Spatiales, Observatoire de Paris-Meudon, F-92195 Meudon Cédex Bitnet: thencre (a) frmeu51

16 - 29

Crieff, Scotland, UK

NATO Adv. Study Inst.

The Sun: A Laboratory for Astrophysics

J.T. Schmelz, SMM-XRP Code 602.6, NASA/Goddard Space Flight Center, Greenbelt, MD 20771, USA - I ++1 (301) 2204171 solmax@jts

16 - 29

St. Andrews, Scotland

39th Scottish Univ. Summer School: High Temperature Superconductivity - Materials, Mechanisms and Devices

J.T.S. Irvine, Chemistry Dept., Univ. of Aberdeen, Aberdeen, AB9 $2 \mathrm{UE}, \mathrm{UK}$

++44 (224) $272924 / 272921$

j.irvine @ uk.ac.abdn

A: 1 Apr $91 /$ PP / 90 / £420. -, incl, board, lodge, incl. proc. / NATO

16 - 30

nt. School of Atomic and Molecular Spectroscopy - 10th Course: Optical Properties of Excited States in Solids

B. DiBartolo, Dept. of Physics, Boston College, Chestnut Hill, MA 02167-3811, USA

$++1(617) 5523575 /$

Ab: 3 Apr 91 / PP / \$US 300.-, incl, board, lodge, incl. proc. / NATO

$17-29$

La Rábida (Huelva), Spain

a Rábida Int. Summer School: Nuclear Physics at the Borderlines

J.M. Arias, Dept. de Fisica Atomica, Fac. de Fisica, Aptdo. 1065, E-41080 Sevilla ++34 (5) $4616615 / 4612097$ Bitnet: ariasc@cica.es

A: 15 Apr 91 / DM 1100.-, incl. board, lodge

17 June - 9 Aug

Trieste, Italy

Summer School in High Energy Physics and Cosmology Int. Centre for Theo. Physics, POB 586, 1-34100 Trieste A: 15 Jan 91

17 June - 27 Sept.

Trieste, Italy

Res. Workshop in Condensed Matter, Atomic and 
22 - 24 June

Summer School on Organic Materi G. Zerbi, Dipt. Chim. Ind., Politecnico

Piazza L. Da Vinci, 32, 1-20133 Milan

++39 (2) $23993235 / 2362589$

darstellung@ imicl64

A: 31 Mar 91 / limited to 100 / ECU 700. - :

350. - students / MPI, CNR

25 June - 5 July Varenna (Como), Italy

nt. School of Physics "Enrico Femi": 67th Course Semiconductor Superlattices and Interfaces

L. Miglio, Dipt. di Fisica, via Celoria, 16, I-20133 Milano ++39 (2) $2392408 / 2392414$

miglio @ milano.infn.it / miglio @ vaxmi

A: 1 May 91 / PP / 50 / inv. / LIT 1500000.-

incl. board, lodge / SIF, CNR, NATO, CEC

30 June - 26 July Les Houches, France

NATO Adv. Study Inst.: Particles in the Nineties

J. Zinn-Justin, Les Houches,

Ecole d'Eté de Physique Théorique,

Côte des Chavants, F-74310 Les Houches

++33 () $50544069 / 59555325$

A: 1 Mar $90 / \mathrm{PP} / 50$ / French /

FF 3300.-, incl. board, lodge, incl. proc.

July - Aug.

USSR

5th School on Physical Structures, Theory and Binary Geometrophysics

Yu.S. Vladmiriv, Physics Faculty, Moscow State Univ. Leninskiye Gory, SU-117234 Moscow

1 - 12 July

Oxford, UK

Oxford/Berkeley Summer Engineering Programme

1- 2 July MOS Devices

3. 4 July Electrostatic Discharge in IC's

4 July Ind. Applns of Neural Networks

8.9 July Chemical Vapour Deposition \& Polycrystalline Si

10- 11 July Microsensors \& Microactuators

10-12 July Applns of Adv. Metallisation in Microelectronics

M. Doherty, Oxford/Berkeley Summer Engng. Prog.

CPD Unit, Dept. for Continuing Education,

Univ. of Oxford, Oxford, OX1 2JA, UK

$++44(865) 270361 / 270309$

A: 21 Jun 91 / NP / $300 / f 275$. - to 875.incl. proc.

$1-12$

Cambridge, UK

NATO Adv. Study Inst.: Clusters and Superclusters of Galaxies

A.C. Fabian, Inst. of Astronomy, Univ. of Cambridge, Madingly Rd., Cambridge, CB3 OHA, UK

$1-13$

Erice, Italy

nt. School of Materials Science and Technology: 21st Course - Surface and Thin Film Magnetism

M. Landolt, HPF-D2, ETH-Zürich,

$\mathrm{CH}-8093$ Zürich

++41(1) $3772270 / 3716268$

A: 15 May $91 / 70 /$ \$ US 650.-, incl. board, lodge

$1-13$

Erice, Italy

Int. School of Materials Science and Tech.: 22nd Course - Semiconductor Materials and Processing Technologies - The Rôle of Phase Transitions, Defects and Diffusion

E. Rimini, Dipt. di Fisica

57 Corso Italia, 1-95129 Catania

++39 (95) $7195418 / 383023$

A: 1 Jul 91 / PP / \$US 450.-, incl. board, lodge incl. proc. / NATO

$5-6$

Barga, Italy urse on Plasma Technology and Applications (Satellite to ICPIG XX limited to 100

7 - 13

Leipzig, Germany

LISS-91: Leipzig Int. Summer School on Chemical Physics of Free Radical and Polymers

H.K. Roth, Sektion Naturwissenschaften, Technische Hochschule, Postfach 66, 0-7030 Leipzig A: 30 Sep $90 / \mathrm{Ab}: 31 \mathrm{Dec} 90 / \mathrm{PP} /$ DM 580.-, incl. proc

8 - 14

Esztergom, Hungary

Summer School on High Temperature Superconductivity

J. Bankuti, Dept. of Low Temperature Physics, Roland Eötvös Univ., Puskin u. 5-7, H-1088 Budapest ++36 (1) $1187929 / 1180206$ Council of Europe

8 July - 2 Aug.

Trieste, Italy

Miniworkshop on Strongly Correlated Electron Systems

Int. Centre for Theo. Physics, POB 586, 1-34100 Trieste ++39 (40) $22401 / 224163$ limited to 25

9- 19

Varenna, Italy

Int. School of Physics "Enrico Fermi": 68th Course Laser Manipulation of Atoms and lons

E. Arimondo, Dipt. di Fisica dell'Univ., Piazza Torricelli 2 1-56100 Pisa

++39 (50) $45223 / 48277$

Bitnet: pba@ icnucevm

A: 1 May 91 / $50 /$ LIT 1500000.-, incl. proc. SIF, CNR, NATO, CEC
15 - 17 July

Alghero, Sardinia, Italy

NATO Adv. Study Inst.: Microscopic Simulations of Complex Hydrodynamic Phenomena

M. Mareschal, Chimie Physique, CP231,

Univ. Libre de Bruxelles,

Boulevard du Triomphe, B-1050 Bruxelles

++32 (2) $6505788 / 6505113$

Bitnet: ulbg039@bbrnsf11

A: 30 Mar 91 / PP / \$US 800. -, incl. board, lodge, incl. proc.

16 - 27

Cargèse, Corsica, France

NATO Adv. Study Inst.: New Symmetry Principles in Quantum Field Theory

M.F. Hanseler, IESC - Boîte 230, Tour 15, 1er étage 4, place Jussieu, F-75230 Paris Cédex 05 ++33 (1) $43294260 / 43298213$ cargese@ frcpn11

A: 4 Mar 91 / PP / 70 / inv. / French / CNRS

22 July - 2 Aug.

Trieste, Italy

Miniworkshop on Quantum and Classical Many-Body Theory in Condensed Matter Physics

Int. Centre for Theo. Physics, POB 586, I-34100 Trieste ++39 (40) $22401 / 224163$

22 July - 2 Aug. Bad Windsheim, Germany

NATO Adv. Study Inst.: Methods in Computational Molecular Physics

S. Wilson, Rutherford Appleton Lab., Chilton, Oxon, OX11 0QX, UK

22 July - 9 Aug.

Trieste, Italy

Special Group Activity on Atomic Physics

Int. Centre for Theo. Physics, POB 586, 1-34100 Trieste ++39 (40) $22401 / 224163$

23 July - 2 Aug.

Varenna, Italy

Int. School of Physics "Enrico Fermi" : 69th Course Quantum Chaos

G. Casati, Centro di Cultura Scientifica "A. Volta",

Villa Olmo, via Cantoni , 1, 1-22100 Como

++39 (31) $572213 / 573395$

Bitnet: gcasat @ imiclvx

A: 1 May 90 / PP / 50 / LIT 1500000.incl. board, lodge / SIF, CNR, NATO, CEC

29 July - 9 Aug.

St. Andrews, UK

8th Scottish Univ. Summer School: Physics of Nanostructures

I. Robertson, Dept. of Electronic and

Electrical Engineering, Univ. of Glasgow, Glascow, G12 800, UK

++44 (41) 3398855 ext. $6471 / 3304907$

sussp@uk.ac.gla.eng.nano

A: 15 Mar 91 / PP / 70 / inv. / f 425. -, incl. board, lodge, incl. proc. / NATO

30 July - 31 Aug Les Houches, France

trongly Interacting Fermions and High T Superconductivity

J. Zinn-Justin, Les Houches

Ecole d'Eté de Physique Théorique

Côte des Chavants, F-74310 Les Houches

++33 () $50544069 / 59555325$

A: $1 \mathrm{Mar} 90$ / PP / 50 / French / FF 4050. incl. board, lodge, incl. proc. / NATO

31 July - 3 Aug. Garching bei München, Germany

Int. Summer School on Plasma Chemistry

S. Veprek, Tech. Univ. München, Lichtenbergstr. 4 W-8046 Garching/München

++49 (89) $32093624 / 32092727$

A: 30 Apr 91 / DM 1200.-; 500.- students

(1400. - ; 700. - after $30 \mathrm{Apr} 91$ ), incl. board

lodge, incl. proc.
5 - 16 Aug

Stanford, CA, USA

19th SLAC Summer Inst. on Particle Physics: epton Hadron Scattering

J. Hawthorne, SLAC, Bin 62, POB 4349

Stanford, CA 94309 , USA

++1 (415) $9262877 / 9262923$

ssi@slacvm

A: 31 May $91 /$ PP: 1 Oct $91 / 350$

\$US 330.-; 210.- students, incl. proc. / DoE

$12-24$

Cargèse, Corsica, France

NATO Adv. Study Inst.: Guided Wave Non-linear Optics

D.B. Ostrowsky, Lab. de Physique de la Matière

Condensée, Univ. de Nice, Parc Valrose,

F-06034 Nice Cédex

++33 () $93529919 /$

dostro@frnice51

A: 15 Mar 91 / PP / $60 /$ French

17 Aug. - 7 Sept.

Cambridge, UK

UK Inst. for Theoretical High Energy Physicists

.C. Taylor, DAMPT, Silver St., Cambridge, CB3 9EW, UK

++44 (223) $337872 / 337873$

jct1 @ uk.ac.rl.ib

A: 1 Apr 91 / SERC

18 - 30

Maleme, Crete, Greece

NATO Adv. Study Inst.: Equilibrium Structures \& Properties of Surfaces \& Interfaces

A. Gonis, Div. of Chemistry and Materials Science, Lawrence Livermore Nat. Lab. Livermore, CA 94550, USA

$18-30$

Bodrum, Turkey

NATO Adv. Study Inst.: NMR in Modern Technology

G.E. Maciel, Colorado State Univ., Dept. of Chemistry, Fort Collins, CO 80523, USA

Adv. Study Inst.: Physics and Materials Sci. of High Temperature Superconductors - II

R. Kossowsky, 6327 Burchfield Ave.,

Pittsburg, PA 15217, USA

$++1(412) 4214408 /-$

Ab: 15 Feb 91 / NATO

$19-24$

Sart-Tilman, Liège, Belgium

t. Post-University Courses: Physics, Maths

IPUCIPU Secretariat, POB 224, B-1000 Brussels 29

++32 (2) $2114211 /-$

A: 1 Jul $91 / \mathrm{NP} / 300 /$ French

BF 6000.- approx., incl. proc.

19 - 30

Course on Path Integration

Trieste, Italy

Int. Centre for Theo. Physics, POB 586, I-34100 Trieste ++39 (40) $22401 / 224163$

limited

19 Aug, -6 Sept.

College on Singularity Theory ++39 (40) $22401 / 224163$

23 Aug. - 2 Sept.

14th CERN School of Computing

Barnett, CN Division, CERN, CH-1211 Geneva 23

++41 (22) $7673090 / 7677155$

barnett@ cernvm.cern.ch

A: 15 May 91 / NP / inv. / SFR 1500.-, incl. board

26 Aug. - 5 Sept. Piaski/Mikolajki, Poland

22nd Mazurian Summer School on Nuclear Physics: Selected Topics in Nuclear and Astro Physics

K. Delegacz, Soltan Inst. for Nuclear Studies,

ul. Hiza 69, PL-00681 Warsaw

$++48(22) 213829 / 216727$

A: 1 May 91

\section{(1) FACHINFORMATIONSZENTRUM KARLSRUHE \\ Gesellschaft für wissenschaftlich-technische Information mbH}

\section{DATABASE CONF of FIZ Karlsruhe available on STN}

The database CONF contains about 64000 items on conferences in energy, physics, mathematics and technology, space research and chemistry with more than 4000 records of future events, and is updated weekly.

Conference schedules are included as soon as they are received from scientific organizations worldwide.

An effective way to make use of the CONF file is to request a direct access via STN and/or to establish a search profile which can be automatically processed to check the approximately 250 newly added data per week.

A close cooperation with the European Physical Society guarantees a comprehensive overview of national and international conferences in physics.

Please call or write to:

STN International, c/o FIZ Karlsruhe, P. O. Box 2465, D-7500 Karlsruhe 1. Tel.: + +49 (7247) 8085 55; Fax: ++49 (7247) 808666 . 
26 Aug - 7 Sept.

Predeal, Romania

Int. Summer School: New Trends in Nuclear Physics

Inst. for Atomic Physics, POB MG-6,

Bucharest, Romania

11397

A: 1 Aug 91 / Ab: 15 Jul $91 /$ PP

27 Aug. - 6 Sept.

Varenna, Italy

Int. School of Plasma Physics "Piero Caldirola": Diagnostics for Contemporary Fusion Experiments

E. Sindoni, ISPP, Dip. Physica

16 via Celoria, $1-20133$ Milano

++39 (2) $2392267 / 2392205$

sndon (G) milano.infn.m

A: 15 Jun 91 / Ab: 15 Jun 91 / PP: $5 \operatorname{Sep} 91 / 100 /$

LIT 550000. - ; 200000. - students, incl. proc. / ISPP, CEC, ENEA, CNR

30 Aug. - 5 Sept.

Nantes, France

Stage des Professeurs de Physique, Géographie et Biologie Géologie

CNES - Délégation à la Communication,

18, av. Edouard-Berlin, F-31055 Toulouse Cédex ++33 () $61273131 / 61281327$

\section{1 - 13 Sept. \\ Aussois, France \\ Photoprocesses in Transition Metal} Complexes, Biosystems and Other Molecules: Experiment and Theory

E. Kochanski, UPR 139 du CNRS

Lab. de Chimie Théorique,

Univ. Louis Pasteur, BP 296/R8

F-67008 Strasbourg Cédex

$++3388416834 /$ -

A: 1 Mar $91 /$ PP / 100 /FF 4200.-

4000. - IOM's, incl. board, lodge,

incl. proc. / NATO

1 - 14

Avila, Spain

1st EPS Southern School of Physics: Dynamical Processes in Molecula Physics

G. Delgado-Barrio, Inst. de Fisica Fundamental, Serrano 123, E-28006 Madric

++34 (1) $4112962 /-$

A: 1 May 91 / NP / 40 / suS 420 incl. board, lodge / UNESCO, CEC

2-8

Ascona, Switzerland

Int. Summer School on Ferroelectric Ceramics

N. Setter, Lab. de Céramique, Dépt. des Matériaux, EPFL, 34, ch. de Bellerive, $\mathrm{CH}-1007$ Lausanne ++41 (21) $6932961 / 6932935$

PP / 80 / \$US 250.-, incl. proc.

$2-13$

Istanbul, Turkey

irst Balkan Physics School: Accelerator Physics Research and Applications

E. Arik, CERN, PPE Division, $\mathrm{CH}-1211$ Geneva 23 $++41(22) 7673100 /$

$2-13$ A: 31 May $91 / \mathrm{NP} / 45$

Working Party on Surface Phase Transition

Trieste, Italy

Int. Centre for Theo Physics, POB 586, 1-34100 Trieste ++39 (40) $22401 / 224163$

2- 20

Workshop on Materials Science and Physics of Non-Conventional Energy Sources

Int. Centre for Theo. Physics, POB 586, I-34100 Trieste ++39 (40) $22401 / 224163$

A: 31 Jul 91 / CNR, ENEA

$3-10$

Erice, Italy

Int. School of Mathematics "G. Stampacchia" 12 th Course - Applied Mathematics in the Aero space Field

A. Salvetti, Dept. Aerospace Engineering, Univ. degli Studi di Pisa, via Diotisalvi, 2, 1-56126 Pisa $++39(50) 550200 / 500987$ A: 31 Mar 91 / PP / NCR, GNAFA

9- 10

Bristol, UK

Adv. School on Electron Diffraction (Satellite to EMAG '91)

J. Steeds, H.H. Wills Physics Lab., Bristol Univ., Tyndall Ave., Bristol, BS8 1TL, UK

9-14 Krakow, Poland

SSMS: Int. Summer School on Mechanical Spectroscopy (Satellite to ECIFUAS-6)

L.B. Magalas, Acad. of Mining and Metallurgy Inst. of Metallurgy, Al. Mickiewicza 30

PL-30059 Krakow

$++48(12) 333823 / 331014$

A: 30 Mar $91 /$ PP / $140 /$

SUS 350. - ; 220.- students

(380. - ; 240. - after 1 Jun 91), incl. proc

$9-27$

chool on Dynamical Systems

Trieste, Italy

Int. Centre for Theo. Physics, POB 586, 1-34100 Trieste ++39 (40) $22401 / 224163$

A: 31 Jan 91 / limited

\section{0 - 20 Sept. \\ Skalský dvur \\ 9th Summer School on Computing Techniques in Physics: Numerical Techniques \& Parallelism in Physics \\ J. Nadrchal, Inst. of Physics, \\ Czechoslovak Acad. of Sciences CSAV, \\ Cukrovarnicka 10, CS-162 O0 Praha 6 \\ ++42 (2) $355500 / 3123184$ \\ Ab: 31 Mar 91 / PP / limited to 120 / \\ SFR 300.- ; 280.- IOM's, incl. proc.}

\section{0 - 20}

Maria Laach, Germany

D. Dahmen, Fachbereich Physik,

Postfach 101240, W-5900 Siegen

++49 (271) $7404128 / 7402330$

Ab: 15 May 91 / PP / 55 / German /

DM 400. -, incl. board, lodge / BMFT

$13-19$

Dagomys, USSR

2nd. Int. Youth School on Plasma Physics and Controlled Fusion

A.S. Chuvatin, Kurchatov Inst. of Atomic Energy,

SU-123182 Moscow

++7 (095) $1961635 / 9430073$

A: 1 Mar $91 / 70$

$15-26$

Adv. Study Inst.: Molecular Liquids

New Perspectives in Physics and Chemistry

J.J.C. Teixeira-Dias, Chem. Dept., Univ. of Coimbra, P-3049 Coimbra

$15-29$

Int. School of Particle Physics: Open Issues in the Standard Mode

D. Levaillant, LPC - Collège de France,

11. pl. Marcelin Berthelot, F-75231 Paris Cédex 05

- I ++33 (1) 43546989

A: 10 Jun 91 / limited to 50 /

SFR 1100 - incl board lodge

16 - 19 Carcans-Maubuisson, France

Summer School on Laser Ablation of Electronic Materials: Basic Mechanism \& Applications

P. Siffert, E-MRS, C.T.T., BP 20 ,

F-67037 Strasbourg Cédex

++33 () $88286543 / 88280990$ PP / E-MRS

16 - 21

Maubuisso
Int. Joliot-Curie de Physique Nucléaire: Le Noyau en Pleines Formes

S. Gozlan, In2P3, 20, rue Berbier du Mets,

F-75013 Paris

++33 (1) $433677100 / 43368631$

PP / 70 / French only / FF 1600.-, incl. board, lodge, incl. proc.

16 - 24

Bodrum, Turkey in Physics

N. Erduran, Univ. of Istanbul, Sci. Faculty,

Physics Dept., TR-34459 Istanbul-Vezneciler

++90 (1) $5526123 /-$

Bitnet: fen01@trumvm11

A: 31 May 91 / NP / 50 / \$US 400.-, incl. board

16- 27 Noordwijkerhout, The Netherlands

Adv. Accelerator Physics: CERN Accelerator School and NIKHEF-H

S. von Wartburg, CERN Accelerator School,

SL Division, $\mathrm{CH}-1211$ Geneva 23

casnik @ cernvm.cern.ch

$19-22$

Workshop On Thin GaAs Layers With An Outlook To Other III-V Compounds

B. Szentpali, Res. Inst. for Technical Physics,

Hungarian Acad. of Sciences,

POB 76, $\mathrm{H}-1325$ Hungar

++36 (1) $1691472 / 1698037$

$19-24$

Int. Seminars on Planetary Emergencies

Erice, Italy

G. Coletti, Dipt di Mathematica, Univ.

via Vanvitelli, 1, 1-06100 Perugia

++39 (75) $5855001 / 5852067$

giulicol@ ipguniv

A: 31 Dec 90 / \$US 700.-, incl. board, lodge

$20-29$

Int.Summer School of Nuclear Physics: 13th Course $-4 \pi$ High Resolution Gammaray Spectroscopy

A. Faessler, Inst. for Theoretical Physics,

Univ. of Tübingen, Auf der Morgenstelle 14

W-7400 Tübingen 1

++49 (7071) $296370 / 296375$

Bitnet: ptfa001@ dtuzdv5a

A: 1 Aug 91 / PP / 100 / SFR 900.-, incl, board $22-27$ Irsee, Germany

Gordon Res. Conf.: Organic Superconductors

A.M. Cruickshank, Gordon Research Center, Univ. of Rhode Island, Kingston, RI 02881-0801, USA

++1 (401) $7834011 / 7837644$

Bitnet: bcp101 (1) uriacc

\$US 550.-, incl. board, lodge

23 Sept - 5 Oct.

El Escorial, Spain

NATO Adv. Study Inst.: Defects \& Disorder in Crystalline \& Amorphous Solids

C.R.A. Catlow, The Royal Institution, 21 Albermarle St. London, W1X 4BS, UK

30 Sept - 25 Oct.

Trieste, Italy

Workshop on Soil Physics

Int. Centre for Theo. Physics, POB 586, 1-34100 Trieste $++(40) 22401 / 224163$

30 Sept. - 4 Oct.

Ispra (Varese), Italy Sensing Satellite (ERS-1) SAR Data Course

Secretariat Eurocourses, JRC, 1-21020 Ispra ++39 (332) $789819 / 789839$

1 - 8 Oct.

Erice, Italy

Int. School of Astrophysics "D. Chalonge": 1st Course - Current Topics in Astrofundamental Physics

G. Coletti, Dipt. di Mathematica, Univ., via Vanvitelli, 1, 1-06100 Perugia ++39 (75) $5855001 / 5852067$ giulicol@ipguniv

A: 31 Dec 90 / \$US 700.-, incl. board, lodge

$3-10$

- 10 Erice, Italy Acoustic Sensing and Probing via Cassia, 1216, 1-00189 Roma $++39(6) 3765757 / 3765341$ A: 15 Sep 91 / PP / 60 / \$US 500.-, incl. board, lodge, incl. proc.

$7-11$

Trieste, Italy

Workshop on Stochastic and Deterministic Models

Int. Centre for Theo. Physics, POB 586, 1-34100 Trieste A: 31 Mar 91

7 - 18 (to be confirmed)

2nd Int. Workshop on Radon Monitoring in Radioprotection and Earth Science

Int. Centre for Theo. Physics, POB 586, 1-34100 Trieste ++39 (40) $22401 / 224163$

7 Oct. - 1 Nov. Trieste, Italy

College on Microprocessor-based Real-Time Control: Principles \& Applications in Physics

Int. Centre for Theo. Physics, POB 586, 1-34100 Trieste ++39 (40) $22401 / 224163$ A: 31 May $91 /$ limited

14 Oct. - 8 Nov.

Trieste, Italy

Training College on the Applications of Synchrotron Radiation

Int. Centre for Theo. Physics, POB 586, 1-34100 Trieste ++39 (40) $22401 / 224163$

20 Oct. - 1 Nov.

Int. School of Biophysics: 20th Course -

Erice, Italy Bioelectrochemistry

G. Coletti, Dipt. di Mathematica, Univ. via Vanvitelli, 1, 1-06100 Perugia ++39 (75) $5855001 / 5852067$ giulicol @ ipguniv

A: 31 Dec $90 /$ \$ US 700.-, incl. board, lodge

$21-25$

Ispra (Varese), Italy

Ocean Colour: Theory and Application in the Coastal Zone Scanner Experience Over the Last 10 Years Secretariat Eurocourses, JRC, 1-21020 Ispra ++39 (332) $789819 / 789839$

4- 22 Nov.

3rd Workshop on Telematics

Trieste, Italy

Int. Centre for Theo. Physics, POB 586, 1-34100 Trieste ++39 (40) $22401 / 224163$

A: 30 Apr 91 / limited

18 - 21 (unconfirmed)

Trieste, Italy

Workshop on the Programme on Training \& 